TRANSACTIONS OF THE

AMERICAN MATHEMATICAL SOCIETY

Volume 354, Number 7, Pages 2865-2895

S 0002-9947(02)02952-5

Article electronically published on March 7, 2002

\title{
A PRIORI DEGENERACY OF ONE-DIMENSIONAL ROTATION SETS FOR PERIODIC POINT FREE TORUS MAPS
}

\author{
JAROSLAW KWAPISZ
}

\begin{abstract}
Diffeomorphisms of the two torus that are isotopic to the identity have rotation sets that are convex compact subsets of the plane. We show that certain line segments (including all rationally sloped segments with no rational points) cannot be realized as a rotation set.
\end{abstract}

\section{INTRODUCTION}

Basic Notations. The integer lattice $\mathbb{Z}^{d}$ in $\mathbb{R}^{d}$ acts on the $d$-dimensional Cartesian space $\mathbb{R}^{d}$ by translations; the translation by $v \in \mathbb{Z}^{d}$ is denoted by $T_{v}: \mathbb{R}^{d} \rightarrow \mathbb{R}^{d}$. The quotient $\mathbb{T}^{d}:=\mathbb{R}^{d} / \mathbb{Z}^{d}$ is a $d$-dimensional flat torus with the natural projection $\pi_{\mathbb{T}^{d}}: \mathbb{R}^{d} \rightarrow \mathbb{T}^{d}$ serving as a universal covering. We shall be interested in $C^{1}$ diffeomorphisms $f: \mathbb{T}^{d} \rightarrow \mathbb{T}^{d}$ that are $C^{1}$-isotopic to the identity. Let $\operatorname{Diff}_{0}\left(\mathbb{T}^{d}\right)$ be the space of all such maps and $\operatorname{Diff}_{0}\left(\mathbb{R}^{d}\right)$ be the space of their lifts to $\mathbb{R}^{d}$. We mention that any homotopic to the identity diffeomorphism of $\mathbb{T}^{2}$ is automatically isotopic to the identity (see page 328 in [27]) so that $\mathbf{D i f f}_{0}\left(\mathbb{R}^{2}\right.$ ) consists of the diffeomorphism $\tilde{f}: \mathbb{R}^{2} \rightarrow \mathbb{R}^{2}$ that are $\mathbb{Z}^{2}$-equivariant (i.e. $\tilde{f} \circ T_{v}=T_{v} \circ \tilde{f}$ for all $\left.v \in \mathbb{Z}^{2}\right)$.

The maps in $\operatorname{Diff}_{0}\left(\mathbb{T}^{2}\right)$ are commonly used to study systems of three coupled oscillators. We shall not explain this well known connection in detail (see [3] and the references therein) but instead we convey the idea via the paradigm of Huygens's clocks. Imagine Christiaan Huygens coupling three pendulum clocks of his making by placing them on one shelf and taking the readings $x_{n}$ and $y_{n}$ of the second and third clock after $n$ revolutions of the first (mother) clock for $n=0,1, \ldots$ That there is $\tilde{f} \in \operatorname{Diff}_{0}\left(\mathbb{R}^{2}\right)$ such that $\left(x_{n+1}, y_{n+1}\right)=\tilde{f}\left(x_{n}, y_{n}\right)$ is a sensible idealization. The long time behavior of $x_{n}$ and $y_{n}$ can be anywhere from simple to very chaotic depending on $\tilde{f}$ (i.e. the clocks' design) — see e.g. [3]. Our main result roughly says that, if the second clock has a well defined and irrational frequency, then the third clock has a well defined frequency as well.

Theorem 1.1. If $\tilde{f} \in \operatorname{Diff}_{0}\left(\mathbb{R}^{2}\right)$ has the property that an irrational number $\alpha$ exists such that, for any initial point $(x, y) \in \mathbb{R}^{2}$, the iterates $\left(x_{n}, y_{n}\right)=\tilde{f}^{n}(x, y)$ satisfy

$$
\lim _{n \rightarrow \infty} x_{n} / n=\alpha,
$$

Received by the editors January 10, 2001 and, in revised form, August 31, 2001.

1991 Mathematics Subject Classification. Primary 37E45, 37E30.

Partially supported by NSF grant DMS-9970725 and MONTS-190729. 
then there is a number $\beta$ (rational or irrational) such that

$$
\lim _{n \rightarrow \infty} y_{n} / n=\beta
$$

for all $(x, y) \in \mathbb{R}^{2}$.

When $x_{n}=x_{0}+n \alpha, \tilde{f}$ is a skew-product, and the theorem follows rather easily from the ergodic averaging over the circle rotation by $\alpha$ 13, 16] - see also [21. 32]. (In particular, the irrationality of $\alpha$ is clearly essential.) At the same time, such real-analytic skew-products $f$ exist with $\alpha$ and $\beta$ independent over $\mathbb{Q}$ that are not uniquely ergodic (see $4.5[13]^{1}$ ). This shows that the dynamics of $f$ may be nontrivial and that the obvious generalization of the theorem to the higher dimensions fails. Indeed, let $\tilde{f}: \mathbb{R}^{2} \rightarrow \mathbb{R}^{2}$ be a lift of such an exotic example with two different ergodic measures $\mu_{1}$ and $\mu_{2}$, and pick a continuous $\phi: \mathbb{T}^{2} \rightarrow \mathbb{R}$ with $\int \phi d \mu_{1} \neq \int \phi d \mu_{2}$. For the map $(x, y, z) \mapsto\left(\tilde{f}(x, y), z+\phi\left((x, y) \bmod \mathbb{Z}^{2}\right)\right)$, which is in $\operatorname{Diff}_{0}\left(\mathbb{R}^{3}\right)$, we have $\lim _{n \rightarrow \infty} x_{n} / n=\alpha$ and $\lim _{n \rightarrow \infty} y_{n} / n=\beta$ regardless of the initial condition $\left(x_{0}, y_{0}, z_{0}\right)$, while $\lim _{n \rightarrow \infty} z_{n} / n$ fails to have a common value: it equals $\int \phi d \mu_{i}$ for $\mu_{i}$-typical $\left(x_{0}, y_{0}, z_{0}\right), i=1,2$.

In order to further connect with existing literature, let us recast our result in a slightly more general form. Recall the rotation set $\rho(\tilde{f})$ of $\tilde{f} \in \operatorname{Diff}_{0}\left(\mathbb{R}^{d}\right)$,

$$
\rho(\tilde{f}):=\bigcap_{n=1}^{\infty} \operatorname{cl}\left(\bigcup_{k=n}^{\infty}\left\{\frac{\tilde{f}^{k}(p)-p}{k}: p \in \mathbb{R}^{d}\right\}\right) \text {. }
$$

This is to say that $\rho(\tilde{f})$ collects all the limits points of the sequences of the form $\frac{\tilde{f}^{k_{i}}\left(p_{i}\right)-p_{i}}{k_{i}}$ where $k_{i} \rightarrow \infty$ and $p_{i} \in \mathbb{R}^{d}$. (The $\mathbb{R}^{d}$ containing $\rho(\tilde{f})$ is rightfully thought of as the real homology group $H_{1}\left(\mathbb{T}^{d}\right)$ but we suppress this distinction, cf. 9].) The set $\rho(\tilde{f})$ is always compact and connected [28. In dimension $d=2$, $\rho(\tilde{f})$ is also convex 28 and much is known about its relation with the dynamics [6, 29] 25, 19, 17, yet it is still open what convex sets can be realized as rotation sets [18, 20]. Thanks to Theorem 1.1] we now know that the vertical segments over an irrational number are precluded. The following is an easy generalization obtained by conjugation and flow-equivalence (see Section 2).

Theorem 1.2. Suppose that $\tilde{f} \in \operatorname{Diff}_{0}\left(\mathbb{R}^{2}\right)$ and the rotation set $\rho(\tilde{f})$ is contained in a line. If either of the two conditions below holds:

(i) the line has rational slope and contains no rational points,

(ii) the line contains a unique rational point and that point is not in $\rho(\tilde{f})$,

then the rotation set $\rho(\tilde{f})$ is just a single point. (Here by a rational point we mean a point with both coordinates that are rational numbers.)

For comparison, let us paraphrase the conjecture of Franks and Misiurewicz inspired by the complete classification of the rotation sets for toral flows in [10].

Conjecture 1 (Franks and Misiurewicz). Suppose that $\tilde{f} \in \operatorname{Diff}_{0}\left(\mathbb{R}^{2}\right)$ is such that the rotation set $\rho(\tilde{f})$ is contained in a line and either

(a) the line contains no rational points, or

(b) the line contains a unique rational point, which is not an endpoint of $\rho(\tilde{f})$,

\footnotetext{
${ }^{1}$ This example arises as the projective action of a $\mathbf{S L}_{2}(\mathbb{R})$-cocyle with positive Lyapunov exponent but no hyperbolic structure. Proposition 3.1 in [15] assures that there is more than one invariant measure.
} 
then the rotation set $\rho(\tilde{f})$ is just a single point.

Thus the two possibilities that remain open are when the line has irrational slope and either misses all rational points (case (a)) or contains exactly one rational point that sits strictly inside the segment $\rho(\tilde{f})$ (case $(\mathrm{b})$ ). Ruling out the first possibility is particularly nagging as it would show that a periodic point free and area preserving 2 $f \in \operatorname{Diff}_{0}\left(\mathbb{T}^{2}\right)$ has a well defined rotation vector, i.e. $\rho(\tilde{f})$ is a point. This can be deduced because such maps have no rational points in their rotation sets ([6, 8] see also $12,5,17]$ ). We hope that our methods will ultimately lead to a resolution of the problem, and we offer Theorem 1.2 as a step in that direction. Let us also comment that the $C^{1}$-regularity required of $f$ is most likely an excessive assumption: our key estimates depend on mere quasiconformality of $f$. Homeomorphic counterexamples presumably exist although they may be quite elusive.

Our arguments break up into two parts: topological and analytical. The topological part hinges on the following result reminiscent of the Brouwer plane translation theorem (cf. [31 7] and the references therein).

Theorem 1.3 (Translation Loop Theorem). Suppose that $f \in \operatorname{Diff}_{0}\left(\mathbb{T}^{2}\right)$ and $\tilde{f} \in$ $\operatorname{Diff}_{0}\left(\mathbb{R}^{2}\right)$ is its lift such that the rotation set $\rho(\tilde{f})$ is contained in a line $L$ with rational slope and no rational points, and let $L_{0}$ be the line through the origin parallel to $L$. Then, for any $n \in \mathbb{N}$, there is a smooth closed simple loop $\gamma$ in $\mathbb{T}^{2}$ - called a translation loop - that is homologous to the loop $\lambda:=L_{0}\left(\bmod \mathbb{Z}^{2}\right)$ and such that $\gamma, \ldots, f^{n}(\gamma)$ are disjoint. Moreover, the ordering of $\gamma, \ldots, f^{n}(\gamma)$ on $\mathbb{T}^{2}$ is the same as that of $\lambda, \ldots, T^{n}(\lambda)$ where $T$ is a translation of $\mathbb{T}^{2}$ that maps $L_{0}$ to $L$.

In the context of Theorem $1.1 \gamma$ is a $(0,1)$-loop on $\mathbb{T}^{2}$ and $\gamma, \ldots, f^{n}(\gamma)$ are ordered on $\mathbb{T}^{2}$ in the same way as $0, \alpha, \ldots, n \alpha$ are ordered on $\mathbb{T}$ - see Figure 1.1. Furstenberg's classical skew-product example [26], which is of the form $\tilde{f}:(x, y) \mapsto$ $(x+\alpha+g(y), y+\beta)$ with $\rho(\tilde{f})=(\alpha, \beta)$, demonstrates that there may be no one $\gamma$ such that $f^{n}(\gamma) \cap \gamma=\emptyset$ for all $n \in \mathbb{N}$. The reason is that as $n$ increases to $\infty, f^{n}(\gamma)$ gets smeared all over $\mathbb{T}^{2}$ so that $f^{n}(\gamma)$ has to eventually intersect any fixed $(0,1)$-loop. This highlights the fact that $f$ need not be semi-conjugated to the irrational circle rotation $R_{\alpha}: \mathbb{T} \rightarrow \mathbb{T}$. Nevertheless, our theorem shows that there is no combinatorial obstruction and such approximate semi-conjugacies exist in the following sense (resembling Rohlin's periodic approximation). Let $\mathrm{p} / \mathrm{q}<$ $\alpha<\mathrm{p}_{*} / \mathrm{q}_{*}$ be a pair of Farey neighboring fractions approximating $\alpha$, and let $\gamma$ be the translation loop for $n=\mathrm{q}+\mathrm{q}_{*}-1$. Denote by $J$ the annulus bounded by $\gamma$ and $f^{\mathrm{q}}(\gamma)$ and by $J_{*}$ the annulus bounded by $\gamma$ and $f^{\mathrm{q}_{*}}(\gamma)$. By the standard combinatorics of $R_{\alpha}$, the family

$$
J, \ldots, f^{\mathrm{q}_{*}-1}(J), J_{*}, \ldots, f^{\mathrm{q}-1}\left(J_{*}\right)
$$

covers all of $\mathbb{T}^{2}$ without overlap (Figure 1.1). It is now easy to produce $h: \mathbb{T}^{2} \rightarrow \mathbb{T}$ so that $R_{\alpha} \circ h(p)=h \circ f(p)$ for $p \notin f^{\mathbf{q}_{*}-1}(J) \cup f^{q-1}\left(J_{*}\right)$ by extending (via dynamics) any continuous map $J \cup J_{*} \rightarrow\left[R^{\mathrm{q}}(0), R^{\mathrm{q}_{*}}(0)\right]$ that sends $f^{\mathrm{q}}(\gamma), \gamma$, and $f^{\mathrm{q}_{*}}(\gamma)$ to $R^{\mathrm{q}}(0)$, 0 , and $R^{\mathrm{q}_{*}}(0)$, correspondingly. Such $h$ nearly semi-conjugates $f$ to $R_{\alpha}$ in the sense that $\max _{p \in \mathbb{T}^{2}} \operatorname{dist}\left(R_{\alpha} \circ h(p)-h \circ f(p)\right) \leq\left|\mathrm{p} / \mathrm{q}-\mathrm{p}_{*} / \mathrm{q}_{*}\right|$.

From another point of view the translation loop theorem is a result on existence of renormalizations. The boundary curves of $J \cup J_{*}$ are naturally identified by $f^{\mathrm{q}-\mathrm{q}_{*}}$

\footnotetext{
${ }^{2}$ The Reeb flow shows that the measure preservation assumption is necessary (see e.g. 28]).
} 


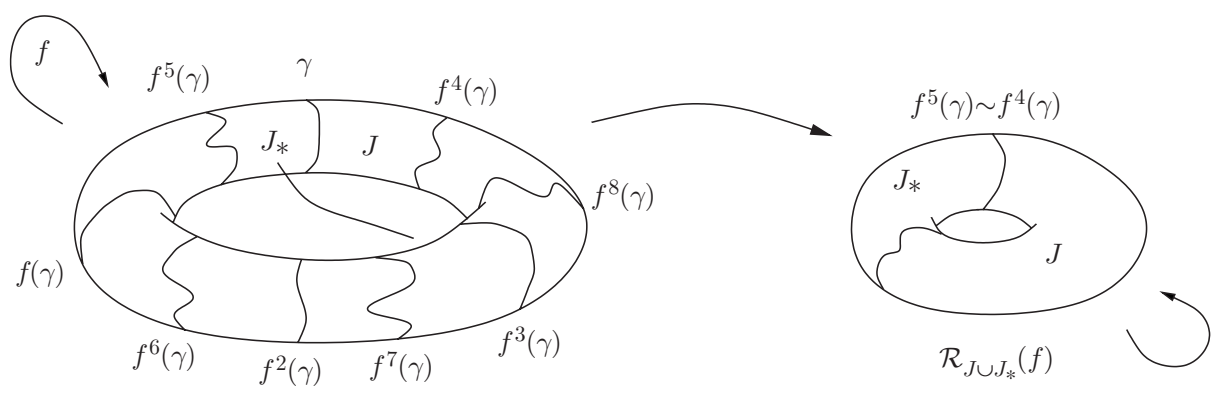

Figure 1.1. Translation loop and renormalization $(\mathrm{p} / \mathrm{q}=4 / 5$, $\left.\mathrm{p}_{*} / \mathrm{q}_{*}=3 / 4\right)$.

so that $\left(J \cup J_{*}\right) /\left(f^{q_{*}}(p) \sim f^{\mathrm{q}}(p)\right) \cong \mathbb{T}^{2}$, and the return map to $J \cup J_{*}$ descends to a homeomorphism $\mathcal{R}_{J \cup J_{*}}(f):\left(J \cup J_{*}\right) / \sim \rightarrow\left(J \cup J_{*}\right) / \sim$. Conjugated via some (normalizing) homeomorphism $\eta:\left(J \cup J_{*}\right) / \sim \rightarrow \mathbb{T}^{2}, \mathcal{R}_{J \cup J_{*}}(f)$ is again a map in $\operatorname{Diff}_{0}\left(\mathbb{T}^{2}\right)$ and constitutes a renormalization of $f$. In contrast to the renormalization described in 22, this procedure engages only one coordinate of the rotation set and also applies to annulus maps (with a well defined rotation vector). Section 5 contains more details; however, a more systematic study of renormalization and a discussion of its position among other existing renormalization schemes is relegated to [23].

Continued fraction approximation $p_{k} / q_{k} \rightarrow \alpha$ yields a whole sequence of the associated renormalizations and raises a general question: Under what circumstances, the renormalizations converge, or are otherwise well behaved in some precisely defined sense? A sufficiently strong answer may as well secure conjugacy to the translation (but will not come easily, cf. [14]). A very weak answer, regarding only the conformal modulus of the renormalized tori, already suffices for the analytical part of the proof of Theorem 1.1 Concretely, the subadditive property of the conformal modulus shall guarantee that $\sum_{j=0}^{\mathbf{q}_{*}-1} \bmod \left(f^{j}(J)\right)+\sum_{j=0}^{\mathbf{q}-1} \bmod \left(f^{j}\left(J_{*}\right)\right) \leq C$ where $C$ is a universal constant (and $\bmod (A)$ stands for the modulus of the annulus $A$ in $\mathbb{T}^{2} \simeq \mathbb{C} /(\mathbb{Z}+i \mathbb{Z})$ ). This roughly means that the annuli of the partition (1.2) get thin as $\mathbf{q}$ and $\mathbf{q}_{*}$ become large so that $f$ resembles in some sense a skew-product of circle homeomorphism 3 - for which the rotation set is a point, as we have already mentioned. This is of course a caricature of the actual proof and the success is not immediately obvious, if only because "conformally thin" is not necessarily "geometrically thin". We shall uniformize the larger annuli $f^{j}\left(J \cup J_{*}\right)$ to $A_{j}:=\mathbb{T} \times\left[0, a_{j}\right] \subset \mathbb{C}$ and then consider the open cascade of mappings $f_{j}: A_{j} \rightarrow A_{j+1}$ induced by $f$. The family $\left\{f_{j}\right\}_{j \in \mathbb{N}}$ generally fails to be uniformly $C^{1}$ due to the unbounded geometry of $f^{j}(\gamma)$ - cf. Furstenberg's example - yet $\left\{f_{j}\right\}_{j \in \mathbb{N}}$ is manifestly uniformly quasiconformal. This facilitates the use of the quasi-invariance of the extremal length in order to bound the displacement of $f_{j}$ 's and to control the geometry of $f^{j}\left(J \cup J_{*}\right)$ 's - see Section 6.

Let us also briefly comment on the proof of the translation loop theorem. We study the suspension flow $\phi$ on $\mathbb{T}^{2} \times \mathbb{T} \simeq \mathbb{T}^{3}$ for which the translation loop corresponds to the $(0,1,0)$-loop $\Gamma$ in $\mathbb{T}^{3}$ such that $\phi^{t}(\Gamma) \cup \Gamma=\emptyset$ for $t \in[0, n]$. The key idea is to seek $\Gamma$ as the intersection of a pair of global cross-sections $\Sigma$ and $\Sigma_{*}$ (both

${ }^{3}$ The renormalizations degenerate to 1 -dimensional maps. 
being 2-tori). In the simplest case, such cross-sections are flat and lift to planes in $\mathbb{R}^{3}$. The complement of the two planes in $\mathbb{R}^{3}$ constitutes four wedges, and at least one wedge is forward flow-invariant so that it contains the full forward orbit of the line of intersection of the two planes, which is a lift of $\Gamma$ (cf. Figure 4.1). Now, the narrower and more irrationally sloped the wedge, the longer $\Gamma$ flows without intersecting its initial position, thus securing a substantial translation time $(n)$. Of course, flat cross-sections may be unattainable, and we use the following topologically invariant definition to single out the pairs of cross-sections that generate translation loops.

Definition 1.1. A pair $\left(\Sigma, \Sigma_{*}\right)$ of embedded (and cohomologically different) tori in $\mathbb{T}^{3}$ is called clean if the tori intersect transversally along a single closed loop. If, additionally, $\Sigma$ and $\Sigma_{*}$ are cross-sections to a flow, then the pair $\left(\Sigma, \Sigma_{*}\right)$ is called a clean pair of cross-sections.

In this way, the key to the translation loop theorem is existence of an appropriate clean pair of cross-sections. Here, the individual cross-sections are readily supplied by the classical result of Fried [11, and the bulk of the work is devoted to isotoping them into a clean pair. The following general question transcends the narrow context of our arguments: Under what circumstances, can one adjust by isotopy a collection of global cross-sections to a flow on a manifold so that their mutual intersections are the simplest possible topologically? In [22], we give a positive answer for triples of cross-sections to a flow on $\mathbb{T}^{3}$ and, as a result, we discover the combinatorics of maps in $\operatorname{Diff}_{0}\left(\mathbb{T}^{2}\right)$ that have a well defined and non-resonant rotation vector. Apparently, the idea of exploiting the flow to shed light on the map (and not vice versa, as pioneered by Poincaré) has a lot to offer and goes beyond its simplest incarnation as the classical flow equivalence. At the same time, our arguments can and should be distilled into purely two-dimensional proofs - if only for the sake of freeing them from intuitive yet non-trivial geometric topology in $\mathbb{R}^{3}$ used in Section 3. We have restrained from doing so to preserve the simplicity of the original geometric ideas.

Let us finish with a quick outline of what follows. A preliminary Section 2 reviews the interplay between rotation sets and flow-equivalence (reducing Theorem 1.2 to Theorem 1.1 in the process). Sections 3 and 4 demonstrate the translation loop theorem. Section 5 is a digression devoted to renormalization of torus and annulus maps. Section 6 contains the analytical estimates that combine with the translation loop theorem to establish Theorem 1.1. Section 7 gathers the general extremal length estimates needed in Section 6. Finally, Appendix 1 supplies some classical yet hard to find geometric topology needed in Sections 2 and 3.

\section{Preliminaries on Flow Equivalence}

In this preliminary section, we review the interplay between the maps in $\operatorname{Diff}_{0}\left(\mathbb{T}^{2}\right)$ and flows on $\mathbb{T}^{3}$ with Fried's [11] serving as our main source. We also reduce Theorem 1.2 to Theorem 1.1

For concreteness, we identify the real homology $H_{1}\left(\mathbb{T}^{3}\right)$ with $\mathbb{R}^{3}$ by choosing the basis made of the standard loops (in the coordinate directions). First, we suspend $f \in \operatorname{Diff}_{0}\left(\mathbb{T}^{2}\right)$ into a flow as follows. We embed the $\mathbb{R}^{2}$ acted upon by $\tilde{f}$ in $\mathbb{R}^{3}$ as the $(x, y)$-plane

$$
\tilde{\mathcal{S}}:=\left\{(x, y, 0):(x, y) \in \mathbb{R}^{2}\right\}
$$


and we embed the corresponding two-torus $\mathbb{T}^{2}$ acted upon by $f$ in $\mathbb{T}^{3}$ as

$$
\mathcal{S}:=\pi_{\mathbb{T}^{3}}(\tilde{\mathcal{S}})=\left\{(x, y, 0):(x, y)\left(\bmod \mathbb{Z}^{2}\right) \in \mathbb{R}^{2}\right\} .
$$

We use a smooth isotopy joining identity to $f$ to generate a flow $\phi: \mathbb{R} \times \mathbb{T}^{3} \rightarrow \mathbb{T}^{3}$ whose time-one-map is $f,\left.\phi^{1}\right|_{\mathcal{S}}=f$. There is the (unique) lifted flow $\tilde{\phi}: \mathbb{R} \times \mathbb{R}^{3} \rightarrow$ $\mathbb{R}^{3}$; and the lifts of $f$ are given as $\left.T_{(a, b, 1)}^{-1} \circ \tilde{\phi}^{1}\right|_{\tilde{\mathcal{S}}}$ where $a, b \in \mathbb{Z}$. We may as well assume that

$$
\tilde{f}=\left.T_{(0,0,1)}^{-1} \circ \tilde{\phi}^{1}\right|_{\tilde{\mathcal{S}}}
$$

Now, $\phi$ (as any flow on $\mathbb{T}^{3}$ ) has its rotation set defined as the rotation set of the time-one-map in $\mathbb{R}^{3}, \rho(\phi):=\rho\left(\tilde{\phi}^{1}\right)$. Likewise, the rotation vector of $p \in$ $\mathbb{T}^{3}$ is $\rho(p, \phi):=\lim _{\tau \rightarrow \infty}\left(\tilde{\phi}^{\tau}(\tilde{p})-\tilde{p}\right) / \tau$ computed on any lift $\tilde{p}$ of $p$ (provided the limit exists). The homological direction of $p$ under $\phi$, as defined in [1], is the projectivization $[\rho(p, \phi)]$ of $\rho(p, \phi)$ where

$$
[v]:= \begin{cases}v \cdot \mathbb{R}_{+} & \text {if } v \neq 0, \\ 0 & \text { if } v=0,\end{cases}
$$

is considered an element of the collection of rays in $\mathbb{R}^{3}$ with 0 added as an extra isolated point. The set of homological directions of $\phi$ is $[\rho(\phi)]:=\{[v]: v \in \rho(\phi)\}$. Unlike $\rho(\phi),[\rho(\phi)]$ it is determined already by the oriented foliation of the flow. Since $\phi$ is a suspension of $f, 0 \notin[\rho(\phi)]$ and an easy computation yields

$$
[(\rho(\tilde{f}), 1)]=[\rho(\phi)]
$$

Another basic (and easy) fact is that conjugating $\phi$ to $\psi=h \circ \phi \circ h^{-1}$ via a homeomorphism $h: \mathbb{T}^{3} \rightarrow \mathbb{T}^{3}$ yields

$$
[\rho(\psi)]=A[\rho(\phi)]
$$

where $A \in \mathbf{S L}_{3}(\mathbb{Z})$ is induced by the linear action of $h$ on the homology $H_{1}\left(\mathbb{T}^{3}\right) \cong$ $\mathbb{R}^{3}$.

Now, recall that a cross-section to a flow is a codimension one smooth submanifold that is transversal to the flow and meets its every flow line. A cross-section $\Sigma$ to $\phi$ is of course necessarily diffeomorphic to $\mathbb{T}^{2}$ (because $\Sigma \times \mathbb{R}$ is a $\mathbb{Z}$-cover of $\mathbb{T}^{3}$ and therefore $\mathbb{Z}^{2}$-quotient of $\mathbb{R}^{3}$ ). The classical result of David Fried on existence of cross-sections (Theorem D in [11]) reads in our context as follows.

Theorem 2.4 (Fried). Suppose that $\phi$ is a flow on $\mathbb{T}^{3}$ and we are given an indivisible integer 1 -cohomology class $\alpha, \alpha: \mathbb{R}^{3} \rightarrow \mathbb{R}$. If $\alpha$ is positive on the set of vectors in $[\rho(\phi)]$ (i.e. $\alpha(v)>0$ if $[v] \in[\rho(\phi)]$ ), then $\phi$ has a cross-section $\Sigma$ in the cohomology class of $\alpha$.

Let us relate $[\rho(\phi)]$ and the rotation set of the return map to a cross-section $\Sigma$. Suppose that $\sigma: \mathbb{T}^{2} \rightarrow \mathbb{T}^{3}$ is an embedding and $\Sigma:=\sigma\left(\mathbb{T}^{2}\right)$ is a cross-section to $\phi$. We have the return map $\phi_{\Sigma}: \Sigma \rightarrow \Sigma$ and the induced map $f \in \operatorname{Diff}_{0}\left(\mathbb{T}^{2}\right)$ is

$$
f:=\sigma^{-1} \circ \phi_{\Sigma} \circ \sigma \text {. }
$$

To recover lifts of $f$ from $\tilde{\phi}$, fix some lift $\tilde{\sigma}: \mathbb{R}^{2} \rightarrow \mathbb{R}^{3}$ and set $\tilde{\Sigma}=\tilde{\sigma}\left(\mathbb{R}^{2}\right)$. Let $u, v \in \mathbb{Z}^{3}$ be the images of $(1,0)$ and $(0,1)$ under the map induced by $\sigma$ on the first homology. Let $w \in \mathbb{Z}^{3}$, be such that $\tilde{\Sigma}+w$ is the lift of $\Sigma$ adjacent to $\tilde{\Sigma}$ in the positive flow direction: i.e. $\tilde{\Sigma}+w$ is the connected component of $\pi_{\mathbb{T}^{3}}^{-1}(\Sigma)$ first hit by flow lines departing from $\tilde{\Sigma}$. Let $\tilde{\phi}_{\tilde{\Sigma}}: \tilde{\Sigma} \rightarrow \tilde{\Sigma}+w$ be the holonomy along the 
flow lines: i.e. $\tilde{\phi}_{\tilde{\Sigma}}(p)$ is the (unique) intersection with $\tilde{\Sigma}+w$ of the flow line through $p \in \tilde{\Sigma}$. It is easy to see that, $\tilde{f}: \mathbb{R}^{2} \rightarrow \mathbb{R}^{2}$ given by

$$
\tilde{f}:=\tilde{\sigma}^{-1} \circ T_{w}^{-1} \circ \tilde{\phi}_{\tilde{\Sigma}} \circ \tilde{\sigma}
$$

is a lift of $f$ and that it does not depend on the choice of $\tilde{\sigma}$. (Replacing $w$ by $w+a u+b v, a, b \in \mathbb{Z}$ produces all other lifts of $f$.) We claim that

$$
[(\rho(\tilde{f}), 1)]=A[\rho(\phi)],
$$

where $A=A_{(u, v, w)}$ is induced by the linear mapping sending $(1,0,0),(0,1,0)$, $(0,0,1)$ to $u, v, w$, respectively. For a proof, note that the formula (2.3) reduces the problem to the case when $u=(1,0,0), v=(0,1,0)$, and $w=(0,0,1)$, the case already covered by (2.2).

Putting together Fried's theorem and formula (2.4) yields the following result.

Theorem 2.5. Suppose that $\Lambda \subset \mathbb{R}^{2}$ is a rotation set of some $\tilde{f} \in \operatorname{Diff}_{0}\left(\mathbb{R}^{2}\right)$. If $u, v, w \in \mathbb{Z}^{3}$ are a basis of $\mathbb{Z}^{3}$ over $\mathbb{Z}$ such that $[\Lambda \times\{1\}]$ is contained in the open halfspace bounded by the plane $\operatorname{lin}\{u, v\}$ that contains $w$, then

$$
\Lambda^{\prime}:=A_{(u, v, w)}^{-1}[(\Lambda, 1)] \cap\{(x, y, t): t=1\}
$$

is a rotation set of some $\tilde{f}^{\prime} \in \operatorname{Diff}_{0}\left(\mathbb{R}^{2}\right)$ such that $\tilde{f}$ and $\tilde{f}^{\prime}$ are lifts of flow equivalent torus maps.

Corollary 2.1. Under the assumptions of Theorem [2.5, if $L$ is a projective linear transformation,

$$
L(x, y)=\frac{\left(a_{11} x+a_{12} y+a_{13}, a_{21} x+a_{22} y+a_{23}\right)}{a_{31} x+a_{32} y+a_{33}},
$$

with the coefficient matrix $A=\left(a_{i j}\right)_{i, j=1}^{3}$ in $\mathbf{S L}_{3}(\mathbb{Z})$ and $a_{31} x+a_{32} y+a_{33} \neq 0$ for $(x, y) \in \Lambda$, then $\Lambda^{\prime}:=L(\Lambda)$ is a rotation set of some $\tilde{f}^{\prime} \in \operatorname{Diff}_{0}\left(\mathbb{R}^{2}\right)$.

Reduction of Theorem 1.2 to Theorem 1.1 (via Corollary 2.5). First, suppose $\tilde{f}$ satisfies the hypothesis (i) of Theorem 1.2, i.e. $\Lambda=\rho(\tilde{f})$ is contained in a line $y=s / r \cdot x+c$ where $s / r$ is a reduced fraction. Let $s_{*} / r_{*}$ be a Farey neighbor of $s / r$ so that $r s_{*}-s r_{*}=1$, and take $L(x, y)=\left(s x-r y, s_{*} x-r_{*} y\right)$. Then $\Lambda^{\prime}=L(\Lambda)$ is contained in a vertical line $x=c$ and we are done by invoking Theorem 1.1

Second, suppose that $\tilde{f}$ satisfies the hypothesis (ii) of Theorem 1.2 with $(p, q) / n$ being the only rational point on the line. The rotation set $\Lambda:=\rho\left(T_{(p, q)}^{-1} \circ \tilde{f}^{n}\right)=$ $n \rho(\tilde{f})-(p, q)$ is then contained in a line through origin, and $0 \notin \Lambda$ so that $\Lambda \subset$ $\left\{\lambda \cdot\left(x_{0}, y_{0}\right): \lambda>0\right\}$ for some point $\left(x_{0}, y_{0}\right) \in \mathbb{R}^{2}$. Take $L(x, y):=(x / y,-1 / y)$. Then $\Lambda^{\prime}=L(\Lambda)$ is contained in the line $x=x_{0} / y_{0}$ and we may invoke Theorem 1.1 again.

\section{Existence of Clean Pairs of Cross-Sections}

We fix a $C^{1}$-flow $\phi$ on $\mathbb{T}^{3}$ and its lift $\tilde{\phi}$ on $\mathbb{R}^{3}$. Let $\mathrm{p} / \mathbf{q}$ and $\mathbf{p}_{*} / \mathbf{q}_{*}$ (with $\mathbf{q}, \mathbf{q}_{*}>0$ ) be any two adjacent Farey fractions such that $\mathrm{p}_{*} / \mathrm{q}_{*}<\mathrm{p} / \mathrm{q}$, which is to say that

$$
A=\left(\begin{array}{cc}
\mathrm{p} & \mathrm{p}_{*} \\
\mathrm{q} & \mathrm{q}_{*}
\end{array}\right) \in \mathbf{S L}_{2}(\mathbb{Z}) .
$$

Throughout this section, we assume that the homological directions of $\phi$ are contained in the wedge between $(p, 0, q)$ and $\left(\mathbf{p}_{*}, 0, \mathbf{q}_{*}\right)$ : 
Hypothesis $(\mathbf{H}):[\rho(\phi)] \subset\left[\left\{a \cdot(\mathrm{p}, 0, \mathbf{q})+b \cdot\left(\mathrm{p}_{*}, 0, \mathbf{q}_{*}\right)+c \cdot(0,1,0): a, b, c>0\right\}\right]$. In view of (2.2), $(\mathrm{H})$ holds when $\tilde{\phi}$ is a suspension of $\tilde{f} \in \operatorname{Diff}_{0}\left(\mathbb{R}^{2}\right)$ and $\rho(\tilde{f}) \subset$ $\left(\mathrm{p}_{*} / \mathrm{q}_{*}, \mathrm{p} / \mathrm{q}\right) \times \mathbb{R}$, which is the case under the assumptions of Theorem 1.1 if $\mathrm{p}_{*} / \mathrm{q}_{*}<$ $\alpha<\mathrm{p} / \mathrm{q}$. (In fact, $(H)$ forces that $\tilde{\phi}$ is a suspension flow for some $\tilde{f} \in \operatorname{Diff}_{0}\left(\mathbb{T}^{2}\right)$.)

Considering $\mathbb{R}^{3}$ as the $(x, y, t)$-space, we write $[\omega]_{H^{1}\left(\mathbb{T}^{3}\right)}$ and $\left[\omega_{*}\right]_{H^{1}\left(\mathbb{T}^{3}\right)}$ for the 1-cohomology classes of the 1 -forms

$$
\begin{gathered}
\omega:=\mathbf{q} d x-\mathrm{p} d t, \\
\omega_{*}:=-\mathbf{q}_{*} d x+\mathbf{p}_{*} d t .
\end{gathered}
$$

By hypothesis $(\mathrm{H}),[\omega]_{H^{1}\left(\mathbb{T}^{3}\right)}$ and $\left[\omega_{*}\right]_{H^{1}\left(\mathbb{T}^{3}\right)}$ are positive on $[\rho(\phi)]$ so that Fried's result (Theorem 2.4) assures existence of two global embedded cross-sections to $\phi$ : $\Sigma$ in $[\omega]_{H^{1}\left(\mathbb{T}^{3}\right)}$ and $\Sigma_{*}$ in $\left[\omega_{*}\right]_{H^{1}\left(\mathbb{T}^{3}\right)}$. The goal of this section is to show that $\Sigma$ and $\Sigma_{*}$ can always be adjusted to form a clean pair of cross-sections (as defined in the introduction).

Theorem 3.6 (Efficient Cut). Under the assumption $(H)$ there exists a clean pair of cross-sections $\Sigma$ and $\Sigma_{*}$ in the cohomology classes $[\omega]_{H^{1}\left(\mathbb{T}^{3}\right)}$ and $\left[\omega_{*}\right]_{H^{1}\left(\mathbb{T}^{3}\right)}$, respectively.

Remark 3.1. If $\Sigma$ and $\Sigma_{*}$ are initially transversal to each other, then the theorem will be shown by isotoping only one of the cross-sections $\left(\Sigma\right.$ or $\left.\Sigma_{*}\right)$ so that the other cross-section is left unchanged.

Proof of Theorem 3.6. Let $\Sigma$ and $\Sigma_{*}$ be the cross sections in the right cohomology classes supplied by Fried's result. We fix smooth embeddings $\sigma, \sigma_{*}: \mathbb{T}^{2} \rightarrow \mathbb{T}^{3}$ such that $\Sigma=\sigma\left(\mathbb{T}^{2}\right)$ and $\Sigma_{*}=\sigma_{*}\left(\mathbb{T}^{2}\right)$. We assume that $\sigma$ and $\sigma_{*}$ are orientation preserving when $\mathbb{T}^{2}$ is taken with the standard orientation and $\Sigma$ and $\Sigma_{*}$ are oriented so that $\omega>0$ and $\omega_{*}>0$. (Thus a positively oriented 2-frame tangent to $\Sigma$ together with the vector field of $\phi$ forms a positively oriented 3 -frame in $\mathbb{T}^{3}$.)

By the Thom transversality theorem, a $C^{1}$-small perturbation of $\sigma$ and $\sigma_{*}$ can be arranged so that $\Sigma$ and $\Sigma_{*}$ are transversal. Thus $\Sigma \cap \Sigma_{*}$ becomes a 1-dimensional smooth closed submanifold of $\mathbb{T}^{3}$, and the classification of 1-manifolds asserts that $\Sigma \cap \Sigma_{*}=\Gamma_{1} \cup \ldots \cup \Gamma_{r}$ where each $\Gamma_{i}$ is a smooth embedded circle (a loop).

The embeddings $\sigma, \sigma_{*}$ induce monomorphisms on the homologies, $\sigma_{1}, \sigma_{* 1}: H_{1}\left(\mathbb{T}^{2}\right)$ $\rightarrow H_{1}\left(\mathbb{T}^{3}\right)$, and we have

$$
\begin{aligned}
\sigma_{1}\left(H_{1}\left(\mathbb{T}^{2}\right)\right) & =\operatorname{lin}\{(\mathbf{p}, 0, \mathbf{q}),(0,1,0)\}=\operatorname{ker}(\omega), \\
\sigma_{* 1}\left(H_{1}\left(\mathbb{T}^{2}\right)\right) & =\operatorname{lin}\left\{\left(\mathbf{p}_{*}, 0, \mathbf{q}_{*}\right),(0,1,0)\right\}=\operatorname{ker}\left(\omega_{*}\right) .
\end{aligned}
$$

Thus the homology classes of $\Gamma_{i}$ 's satisfy $\left[\Gamma_{i}\right]_{H_{1}\left(\mathbb{T}^{3}\right)} \in \operatorname{lin}\{(0,1,0)\}$. The preimages of $\Gamma_{i}$ 's on $\mathbb{T}^{2}$ are

$$
\alpha_{i}:=\sigma^{-1}\left(\Gamma_{i}\right), \quad\left[\alpha_{i}\right]_{H_{1}\left(\mathbb{T}^{2}\right)} \in \operatorname{lin}\{(0,1)\}
$$

and

$$
\alpha_{* i}:=\sigma_{*}^{-1}\left(\Gamma_{i}\right), \quad\left[\alpha_{* i}\right]_{H_{1}\left(\mathbb{T}^{2}\right)} \in \operatorname{lin}\{(0,1)\},
$$

where $i=1, \ldots, r$. Moreover, if $\bullet: H_{2}\left(\mathbb{T}^{3}\right) \times H_{2}\left(\mathbb{T}^{3}\right) \rightarrow H_{1}\left(\mathbb{T}^{3}\right)$ is the intersection product, then we have

$$
\sum_{i=1}^{r}\left[\Gamma_{i}\right]_{H_{1}\left(\mathbb{T}^{3}\right)}=\left[\Sigma \cap \Sigma_{*}\right]_{H_{1}\left(\mathbb{T}^{3}\right)}=[\Sigma]_{H_{2}\left(\mathbb{T}^{3}\right)} \bullet\left[\Sigma_{*}\right]_{H_{2}\left(\mathbb{T}^{3}\right)}=(0,1,0),
$$


(where an appropriate orientation is put on $\Gamma_{i}$ 's). In this way, if $r=1$, then $\Gamma_{1}$ is a loop with $\left[\Gamma_{1}\right]_{H_{1}\left(\mathbb{T}^{3}\right)}=(0,1,0)$, making $\left(\Sigma, \Sigma_{*}\right)$ a clean pair of cross-sections. In the rest of this proof, we assume that $r>1$ and show how to isotope $\sigma_{*}$ to diminish $r$ by one without destroying the property that $\Sigma_{*}$ is a cross-section to $\phi$. We shall consider two cases separately.

Case 0: There is $k, 1 \leq k \leq r$, such that $\Gamma_{k}$ is null-homotopic.

Clearly, $\Gamma_{i}$ is null-homotopic in $\mathbb{T}^{3}$ iff $\alpha_{i}$ is null-homotopic in $\mathbb{T}^{2}$. Consider then all the null-homotopic loops $\alpha_{i}$. Each such loop bounds a smooth 2-disc $\Delta_{i}$ in $\mathbb{T}^{2}$, and any two such discs are either disjoint or contained one in another. We may fix $k$ with $\Delta_{k}$ minimal with respect to inclusion so that $\Delta_{k}$ is disjoint from all $\alpha_{i}$ with $i \neq k$. Let also $\Delta_{* k}$ be the 2-disc bounded by $\alpha_{* k}$ in $\mathbb{T}^{2}$. (We do not insist that $\Delta_{* k}$ contains no other null-homotopic $\alpha_{* i}$ 's.) In $\mathbb{T}^{3}$, we have $D:=\sigma\left(\Delta_{k}\right)$ and $D_{*}:=\sigma_{*}\left(\Delta_{* k}\right)$ that are two smoothly embedded (closed) discs that intersect (transversally) along $\Gamma_{k}$. Thus $D \cup D_{*}$ is a smoothly embedded sphere except for the singularity along $\Gamma_{k}$. By a version of Schönflies Theorem (in the appendix) the sphere bounds a 3 -disk $B$ in $\mathbb{T}^{3}$.

The flow is transversal through the union of the 2-dimensional interiors $D^{\circ} \cup$ $D_{*}^{o}=\partial B \backslash \Gamma_{k}$. For specificity, we assume that $\phi$ enters $B$ through $D_{*}^{o}$ (i.e. the normal component of the vector field $\left.\frac{d}{d t}\right|_{t=0} \phi^{t}(p)$ points inside $B$ for all $\left.p \in D_{*}^{o}\right)$. Otherwise, one should use the reversed flow in the following arguments. We claim that, since $\phi$ enters through $D_{*}^{o}$, $\phi$ must exit through $D^{o}$. Indeed, otherwise, $\phi$ enters $B$ through $D^{o} \cup D_{*}^{o}$, which implies that, for $p$ in the interior of $B$, the positive flowline $\left\{\phi^{t}(p)\right\}_{t>0}$ is contained in $B$. It follows that $\rho(p, \phi)=0$, which contradicts $0 \notin \rho(\phi)$ as guaranteed by hypothesis $(\mathrm{H})$.
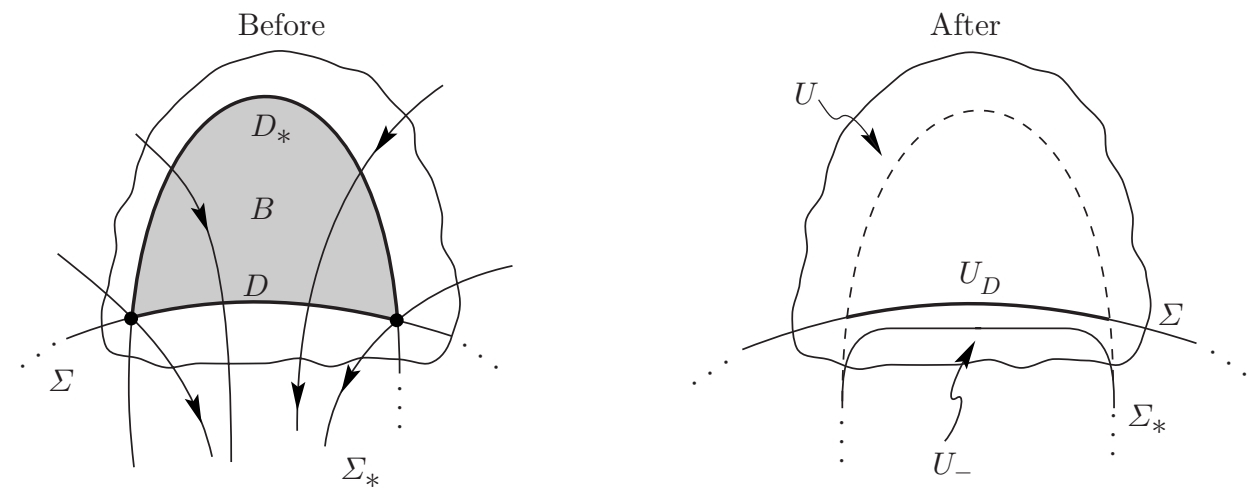

Figure 3.1. Removing a null-homotopic loop from $\Sigma \cap \Sigma_{*}$ (in a cross-cut view).

For an open neighborhood $U$ of $B$ in $\mathbb{T}^{3}$, denote by $U_{D}$ the connected component of $\Sigma \cap U$ that contains $D$. To localize our isotopy, we shall need $U$ satisfying the following conditions (see Figure 3.1):

(i) $U$ is a smoothly embedded open 3 -disk in $\mathbb{T}^{3}$;

(ii) $U_{D}$ is a 2-disk and $U_{D} \cap \Sigma_{*}=\Gamma_{k}$;

(iii) $U_{D}$ cuts $U$ into exactly two connected components of which one, denoted $U^{-}$, is entered by the flow along $U_{D}$;

(iv) $\Sigma_{*} \cap U=D_{*} \cup\left(\Sigma_{*} \cap U^{-}\right)$. 
In fact, for sufficiently small $\epsilon>0$, the mutual transversality of $\Sigma, \Sigma_{*}$, and the flow $\phi$ assures that $U$ equal to the $\epsilon$-neighborhood of $B$ satisfies the above hypotheses (consult Figure 3.1). We skip the routine proof.

We shall now deform $\Sigma_{*}$ inside $U$ as follows. Take $\chi: \mathbb{T}^{3} \rightarrow[0,1]$ to be a smooth (bump) function with $\left.\chi\right|_{U}>0$ and $\left.\chi\right|_{\mathbb{T}^{3} \backslash U}=0$. Let $\psi: \mathbb{R} \times \mathbb{T}^{3} \rightarrow \mathbb{T}^{3}$ be the flow $\phi$ with speed multiplied by $\chi$, i.e. $\left.\frac{d}{d t}\right|_{t=0} \psi^{t}(p)=\left.\chi(p) \cdot \frac{d}{d t}\right|_{t=0} \phi^{t}(p)$ for $p \in \mathbb{T}^{3}$. Clearly, $\psi$ is the identity outside $U$, and the orbit of $\phi$ starting at $p \in U$ must hit $\partial U$ (for otherwise its rotation vector would be zero by (i)). Consider $\psi^{\infty}: \mathbb{T}^{3} \rightarrow \mathbb{T}^{3} \backslash U$ given by $\psi^{\infty}(p):=\lim _{t \rightarrow \infty} \psi^{t}(p)$. (For $p \in U, \psi^{\infty}(p)$ is the first time $\phi^{t}(p)$ hits dU.) We claim that

$$
\psi^{\infty}\left(\Sigma_{*}\right) \cap \Sigma \cap U=\emptyset
$$

(as it is suggested by Figure 3.1). Indeed, from (iii), $\partial U^{-}=\left(\partial U^{-} \cap \partial U\right) \cup U_{D}$, where the flow $\psi$ fixes $\partial U^{-} \cap \partial U$ and enters $U^{-}$through $U_{D}$. Therefore, $U^{-}$is $\psi$-invariant and $\psi^{\infty}\left(U^{-}\right) \subset \partial U^{-} \cap \partial U=\partial U^{-} \backslash \Sigma$; in particular, $\psi^{\infty}\left(U^{-}\right) \cap \Sigma=\emptyset$ so that

$$
\psi^{\infty}\left(U^{-} \cap \Sigma_{*}\right) \cap \Sigma=\emptyset .
$$

At the same time, $\psi^{\infty}\left(D_{*}\right) \subset \psi^{\infty}(D) \subset \psi^{\infty}\left(U^{-}\right)$because if $p \in D_{*}^{o}$ then $\psi^{t}(p)$ moves into $B$ for small $t>0$ only to exit $B$ and enter $U^{-}$through $D$ for some later $t$ (c.f. (iii)). Hence,

$$
\psi^{\infty}\left(D_{*}\right) \cap \Sigma \subset \psi^{\infty}\left(U^{-}\right) \cap \Sigma=\emptyset .
$$

Combining (3.4), (3.5), and the hypothesis (iv) establishes the claim (3.3).

Now, claim (3.3) and (ii) yield

$$
\begin{aligned}
\Sigma \cap \psi^{\infty}\left(\Sigma_{*}\right) & =\left(\Sigma \cap \psi^{\infty}\left(\Sigma_{*}\right) \backslash U\right) \cup\left(\Sigma \cap \psi^{\infty}\left(\Sigma_{*}\right) \cap U\right) \\
& \subset\left(\Sigma \cap \Sigma_{*} \backslash U\right) \cup \emptyset \subset \bigcup_{i=1, i \neq k}^{r} \Gamma_{i} .
\end{aligned}
$$

For large $t_{0}>0$, the above inclusion implies (by compactness) that

$$
\Sigma \cap \psi^{t_{0}}\left(\Sigma_{*}\right) \subset \sum_{i=1, i \neq k}^{r} \Gamma_{i}
$$

Hence, upon replacing $\Sigma_{*}$ with $\psi^{t_{0}}\left(\Sigma_{*}\right)$, the number of connected components of $\Sigma \cap \Sigma_{*}$ drops from $r$ to at most $r-1$; and the modification does not change the cohomology class of $\Sigma_{*}$ because $\psi^{t_{0}} \circ \sigma_{*}: \mathbb{T}^{2} \rightarrow \mathbb{T}^{3}$ is manifestly isotopic to $\sigma_{*}$.

Case 1: All loops $\Gamma_{i}, 1 \leq i \leq r$, are essential (of homology type $\pm(0,1,0)$ ).

In this case all $\alpha_{i}$ 's and $\alpha_{* i}$ 's are of type $\pm(0,1)$. In particular, $\alpha_{i}$ 's cut $\mathbb{T}^{2}$ into annuli. Consider one such annulus $\Delta$ bounded by $\alpha_{i}$ and $\alpha_{j}$. Denote by $\Delta_{*}$ the annulus bounded by $\alpha_{* i}$ and $\alpha_{* j}$. (Note that there may be other $\alpha_{* k}$ 's inside $\Delta_{*}$.) Also, let $A:=\sigma(\Delta)$ and $A_{*}:=\sigma_{*}\left(\Delta_{*}\right)$. Clearly, $A \cap A_{*}=\Gamma_{i} \cup \Gamma_{j}$. Thus $A \cup A_{*}$ is a smoothly embedded 2-torus in $\mathbb{T}^{3}$ except for singularities along $\Gamma_{i}$ and $\Gamma_{j}$. We claim that one can select $\Delta$ so that the embedding sends the fundamental group of $A \cup A_{*}$ to the cyclic subgroup generated by a $(0,1,0)$-loop. The idea - see Figure 3.2 — is to make sure that $\Sigma$ and $\Sigma_{*}$ intersect at $\Gamma_{i}$ and $\Gamma_{j}$ with opposing signs (in the sense of the intersection homology), which can be formalized as follows. Let $\tilde{\Sigma}$ 
and $\tilde{\Sigma}_{*}$ be some connected lifts in $\mathbb{R}^{3}$ of $\Sigma$ and $\Sigma_{*}$, respectively. In view of (3.1), the collection $\pi_{\mathbb{T}^{3}}^{-1}\left(\bigcup_{i=1}^{r} \Gamma_{i}\right)$ of all lifts of the loops $\Gamma_{i}$ can be written as

$$
\begin{aligned}
\left(\tilde{\Sigma}+\mathbb{Z}^{3}\right) \cap\left(\tilde{\Sigma}_{*}+\mathbb{Z}^{3}\right) & =\left(\tilde{\Sigma}+\mathbb{Z}\left(\mathbf{p}_{*}, 0, \mathbf{q}_{*}\right)\right) \cap\left(\tilde{\Sigma}_{*}+\mathbb{Z}(\mathbf{p}, 0, \mathbf{q})\right) \\
& =\tilde{\Sigma} \cap\left(\tilde{\Sigma}_{*}+\mathbb{Z} \cdot(\mathbf{p}, 0, \mathbf{q})\right)+\mathbb{Z}\left(\mathbf{p}_{*}, 0, \mathbf{q}_{*}\right) .
\end{aligned}
$$

Therefore, since $r>1, \tilde{\Sigma} \cap\left(\tilde{\Sigma}_{*}+k(\mathrm{p}, 0, \mathrm{q})\right)$ must have more than one component for some $k \in \mathbb{Z}$. We may well assume that $k=0$ (by an appropriate choice of $\tilde{\Sigma}_{*}$ ). Let then $\tilde{\Gamma}_{i}$ and $\tilde{\Gamma}_{j}$ be two distinct components of $\tilde{\Sigma} \cap \tilde{\Sigma}_{*}$. We may choose $\tilde{\Gamma}_{i}$ and $\tilde{\Gamma}_{j}$ adjacent on $\tilde{\Sigma}$ (in the sense that the strip between $\tilde{\Gamma}_{i}$ and $\tilde{\Gamma}_{j}$ in $\tilde{\Sigma}$ is free of $\tilde{\Sigma}_{*}$ ). The strips bounded by $\tilde{\Gamma}_{i}$ and $\tilde{\Gamma}_{j}$ in $\tilde{\Sigma}$ and $\tilde{\Sigma}_{*}$ project to two annuli $A$ and $A_{*}$, respectively; both bounded by $\Gamma_{i}$ and $\Gamma_{j} . A \cup A_{*}$ is by construction deformable to $\Gamma_{i}$ (or $\Gamma_{j}$ ), which ends the proof of the claim.

In view of the claim, Alexander's theorem on torus embeddings (in the appendix) assures that $A \cup A_{*}$ bounds a solid torus $B \cong \mathbb{T} \times \mathbb{D}^{2}$ in $\mathbb{T}^{3}$.

The flow is transversal through the 2-dimensional interiors

$$
A^{o} \cup A_{*}^{o}=\partial B \backslash\left(\Gamma_{i} \cup \Gamma_{j}\right) .
$$

From this point on the argument is very similar to that in Case 0 - compare Figures 3.1 and 3.2 .

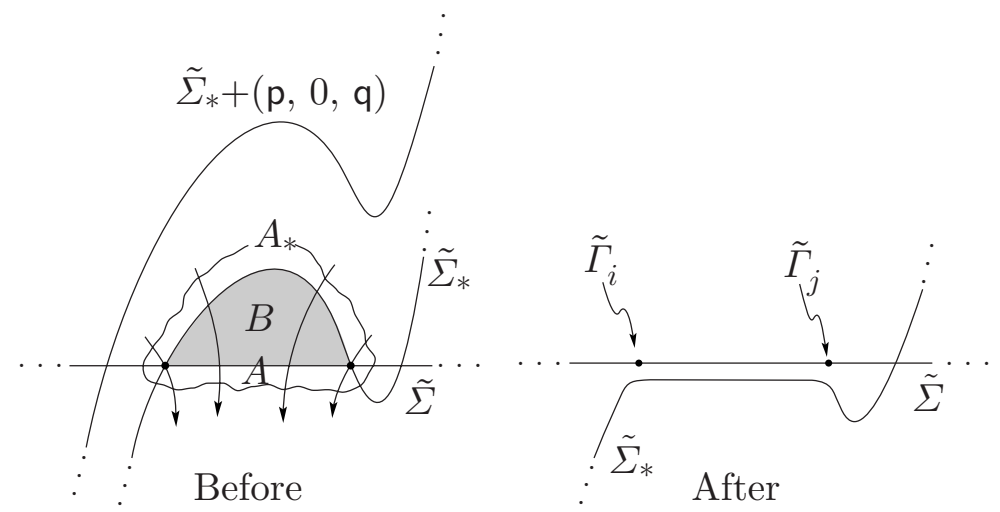

Figure 3.2. Removing a pair of $(0,1,0)$ loops from $\Sigma \cap \Sigma_{*}$

For specificity and at the expense of perhaps reversing the flow, we assume that $\phi$ enters $B$ through $A_{*}^{o}$. We claim that then $\phi$ necessarily exits through $A^{o}$. Indeed, otherwise, $\phi$ enters $B$ through $A^{o} \cup A_{*}^{o}$, which implies that, for $p$ in the interior of $B$, the positive flowline $\left\{\phi^{t}(p)\right\}_{t>0}$ is contained in $B$. Since $B$ deforms onto a $(0,1,0)$-loop in $\mathbb{T}^{3}, \rho(p, \phi) \in \operatorname{lin}\{(0,1,0)\}$ contradicting the hypothesis $(\mathrm{H})$.

Now, for an open neighborhood $U$ of $B$ in $\mathbb{T}^{3}$, let $U_{A}$ be the connected component of $\Sigma \cap U$ that contains $A$. We shall need $U$ satisfying the following conditions:

(i) $U$ is a smooth solid torus that deforms to $B$ in $\mathbb{T}^{3}$;

(ii) $U_{A}$ is an annulus and $U_{A} \cap \Sigma_{*}=\Gamma_{i} \cup \Gamma_{j}$;

(iii) $U_{A}$ cuts $U$ into exactly two connected components, of which one, denoted by $U^{-}$, is entered by the flow along $U_{A}$;

(iv) $\Sigma_{*} \cap U=A_{*} \cup\left(\Sigma_{*} \cap U^{-}\right)$. 
Again, we omit the proof that transversality of $\Sigma, \Sigma_{*}$ and the flow assures that $U$ equal to the $\epsilon$-neighborhood of $B$ satisfies the hypotheses for sufficiently small $\epsilon>0$.

We shall deform $\Sigma_{*}$ inside $U$ to remove $\Gamma_{i} \cup \Gamma_{j}$ from $\Sigma \cap \Sigma_{*}$. Exactly as in Case 0 , take $\chi: \mathbb{T}^{3} \rightarrow[0,1]$ with $\left.\chi\right|_{U}>0$ and $\left.\chi\right|_{\mathbb{T}^{3} \backslash U}=0$; and take $\psi: \mathbb{R} \times \mathbb{T}^{3} \rightarrow \mathbb{T}^{3}$ with $\left.\frac{d}{d t}\right|_{t=0}\left(\psi^{t}(p)\right)=\left.\chi(p) \frac{d}{d t}\right|_{t=0}\left(\phi^{t}(p)\right), p \in \mathbb{T}^{3}$. There is again $\psi^{\infty}: \mathbb{T}^{3} \rightarrow \mathbb{T}^{3} \backslash U$, $\psi^{\infty}(p):=\lim _{t \rightarrow \infty} \psi^{t}(p)$ such that $\psi^{\infty}(p)$ is the first time $\phi^{t}(p)$ hits $\partial U$, as forced for $p \in U$ by the hypothesis $(\mathrm{H})$. We claim that $\psi^{\infty}\left(\Sigma_{*}\right) \cap \Sigma \cap U=\emptyset$. The corresponding proof from Case 0 goes through almost verbatim with $D$ and $D_{*}$ replaced by $A$ and $A_{*}$ - we skip it.

Finally, (ii) and the claim yields

$$
\begin{aligned}
\Sigma \cap \psi^{\infty}\left(\Sigma_{*}\right) & =\left(\Sigma \cap \psi^{\infty}\left(\Sigma_{*}\right) \backslash U\right) \cup\left(\Sigma \cap \psi^{\infty}\left(\Sigma_{*}\right) \cap U\right) \\
& \subset\left(\Sigma \cap \Sigma_{*} \backslash U\right) \subset \bigcup_{k=1, k \neq i, j}^{r} \Gamma_{k} .
\end{aligned}
$$

For large $t_{0}>0$, we have then

$$
\Sigma \cap \psi^{t_{0}}\left(\Sigma_{*}\right) \subset \sum_{k=1, k \neq i, j}^{r} \Gamma_{k} .
$$

The embedding $\psi^{t_{0}} \circ \sigma_{*}: \mathbb{T}^{2} \rightarrow \mathbb{T}^{3}$ is the sought after improvement of $\sigma_{*}$, which reduces the number of connected components of $\Sigma \cap \Sigma_{*}$ by at least two. This ends the description of the induction step and thus finishes the proof of the theorem.

\section{Translation Loop from Clean Intersection}

With a goal of establishing the translation loop theorem (Theorem 1.3), we shall consider now the suspension flow of $f$ together with a clean pair of cross-sections $\Sigma$ and $\Sigma_{*}$ in the cohomology classes of $-\mathrm{q} d x+\mathrm{p} d t$ and $\mathrm{q}_{*} d x-\mathrm{p}_{*} d t$, as constructed in the previous section. The translation loop $\gamma$ will be obtained from the $(0,1,0)$-loop $\Gamma:=\Sigma \cap \Sigma_{*}$ by flowing $\Gamma$ to the original cross-section $\mathcal{S}=\mathbb{T}^{2}$. Recall the guiding idea: the lifts $\tilde{\Sigma}$ and $\tilde{\Sigma}_{*}$ bound a forward invariant wedge $W^{+}$in $\mathbb{R}^{3}$ - cf. Figure 4.1 - so that $\tilde{\Gamma}=\tilde{\Sigma} \cap \tilde{\Sigma}_{*}\left(\right.$ a lift of $\Gamma$ ) satisfies $\tilde{\phi}^{t}(\tilde{\Gamma}) \subset \tilde{\phi}^{t}\left(W^{+}\right) \subset W^{+}$for all $t>0$ and so $\phi^{t}(\Gamma) \cap \Gamma=\emptyset$ for a long time provided $\mathrm{p}, \mathrm{p}_{*}, \mathrm{q}$, and $\mathrm{q}_{*}$ are large (i.e. $W^{+}$is narrow). A more careful analysis of how $W^{+}$projects to $\mathbb{T}^{3}$ will explain the exact ordering of the iterates of $\gamma$.

For a precise formulation, we lift the flow $\phi$, the cross-section $\mathcal{S}$, and $\Gamma$ to the suspension covering $\mathbb{R}^{3} / \Lambda \cong \mathbb{T}^{2} \times \mathbb{R}$ where $\Lambda=\mathbb{Z}(1,0,0)+\mathbb{Z}(0,1,0)$; and we distinguish the corresponding lifted objects by a hat. (The lifts to the universal cover are distinguished by tilde.) In particular, $\hat{\mathcal{S}}$ is an embedded torus pierced by the flow lines of $\hat{\phi}$ in the $t$-direction $(0,0,1)$, and $\hat{\Gamma}$ is a simple loop in $\mathbb{R}^{3} / \Lambda$ of homology type $(0,1)$. Let $\hat{\phi}_{\hat{\mathcal{S}}}: \mathbb{R}^{3} / \Lambda \rightarrow \hat{\mathcal{S}}$ be the projection along the flow lines, i.e. $\hat{\phi}_{\hat{\mathcal{S}}}(p):=\hat{\phi}^{\mathbb{R}}(p) \cap \hat{\mathcal{S}}$ for $p \in \mathbb{R}^{3} / \Lambda$. We define

$$
\hat{\gamma}:=\hat{\phi}_{\hat{\mathcal{S}}}(\hat{\Gamma}) \quad \text { and } \quad \gamma:=\pi_{\mathbb{T}^{3}}(\hat{\gamma})
$$

where $\pi_{\mathbb{T}^{3}}: \mathbb{R}^{3} / \Lambda \rightarrow \mathbb{T}^{3}$ is the natural projection.

Theorem 4.7 (Technical Version of Translation Loop Theorem). If $\tilde{f} \in \mathbf{D i f f}_{0}\left(\mathbb{R}^{2}\right)$ and $\rho(\tilde{f}) \subset\left(\mathbf{p}_{*} / \mathbf{q}_{*}, \mathbf{p} / \mathbf{q}\right) \times \mathbb{R}$ (so that the suspension flow $\phi$ satisfies the hypothesis 
(H) from Section 3), then the projection $\gamma$ of a clean intersection of cross-sections to the torus $\mathcal{S}$ (as defined above) is a smooth simple $(0,1)$-loop in $\mathcal{S}$ with a property that $\gamma, \ldots, f^{\mathrm{q}+\mathrm{q}_{*}-1}(\gamma)$ are disjoint and, for any $\alpha \in\left(\mathrm{p} / \mathrm{q}, \mathrm{p}_{*} / \mathrm{q}_{*}\right)$, there is an orientation preserving homeomorphism $h: \mathbb{T}^{2} \rightarrow \mathbb{T}^{2}$ such that

$$
h\left(\gamma_{j}\right)=\pi_{\mathbb{T}^{2}}\left\{(x, y) \in \mathbb{R}^{2}: x=j \alpha\right\} \text { for } j=0, \ldots, \mathbf{q}+\mathbf{q}_{*}-1 .
$$

The translation loop theorem follows by first conjugating $f$ via a toral automorphism so that the line $\mathcal{L}$ containing the the rotation set becomes $x=$ const (cf. Corollary 2.5) and then applying the theorem above to pairs of Farey fractions $\mathrm{p}_{*} / \mathrm{q}_{*}<\alpha<\mathrm{p} / \mathrm{q}$ that approximate $\alpha$ sufficiently close. The rest of this section is devoted to the proof of Theorem 4.7 .

To ease our exposition, it is convenient to isotope $\mathbb{T}^{3}$ so that $\Sigma$ and $\Sigma_{*}$ become flat tori. This step is facilitated by a general Lemma 8.6 (in the appendix). Moreover, we shall change the basis in $\mathbb{R}^{3}$ (and translate) so that the lifts $\tilde{\Sigma}$ and $\tilde{\Sigma}_{*}$ of $\Sigma$ and $\Sigma_{*}$ become the coordinate planes $\operatorname{lin}\{(1,0,0),(0,1,0)\}$ and $\operatorname{lin}\{(0,0,1),(0,1,0)\}$, respectively. Technically, we are conjugating $\phi$ by the diffeomorphism supplied by the lemma composed with the linear automorphism $L$ induced by $\tilde{L}: \mathbb{R}^{3} \rightarrow \mathbb{R}^{3}$ such that

$$
\tilde{L}=\left(\begin{array}{ccc}
\mathrm{q}_{*} & 0 & -\mathrm{p}_{*} \\
0 & 1 & 0 \\
-\mathrm{q} & 0 & \mathrm{p}
\end{array}\right) \quad \text { and } \quad \tilde{L}^{-1}=\left(\begin{array}{ccc}
\mathrm{p} & 0 & \mathrm{p}_{*} \\
0 & 1 & 0 \\
\mathrm{q} & 0 & \mathrm{q}_{*}
\end{array}\right)
$$

We shall suppress this conjugacy and use the old notation. Figure 4.1 depicts the situation. Note that now $\Lambda=\mathbb{Z}\left(\mathbf{q}_{*}, 0,-\mathbf{q}\right)+\mathbb{Z}(0,1,0)$ and the standard cross-section $\mathcal{S}$ is an embedded (possibly non-flat) torus homologous to the quotient of the plane $\operatorname{lin}\left\{\left(\mathbf{q}_{*}, 0,-\mathbf{q}\right),(0,1,0)\right\}$. The ("time") deck translation, originally $T_{(0,0,1)}$, is now represented by $T_{\mathrm{t}}:=T_{\left(-\mathrm{p}_{*}, 0, \mathrm{p}\right)}$. The (conjugated) flow yields the (conjugated) map $\tilde{f}$ via the analogue of (2.1):

$$
\left.T_{\left(-\mathrm{p}_{*}, 0, \mathrm{p}\right)}{ }^{-1} \circ \tilde{\phi}^{1}\right|_{\tilde{\mathcal{S}}}=\tilde{f} \quad \text { and }\left.\quad T_{\left(-\mathrm{p}_{*}, 0, \mathrm{p}\right)}{ }^{-1} \circ \hat{\phi}^{1}\right|_{\hat{\mathcal{S}}}=\hat{f} .
$$

Also, keep in mind that the positive wedge, $W^{+}=\{(x, y, t): x, t>0\}$, is forward invariant under the flow.

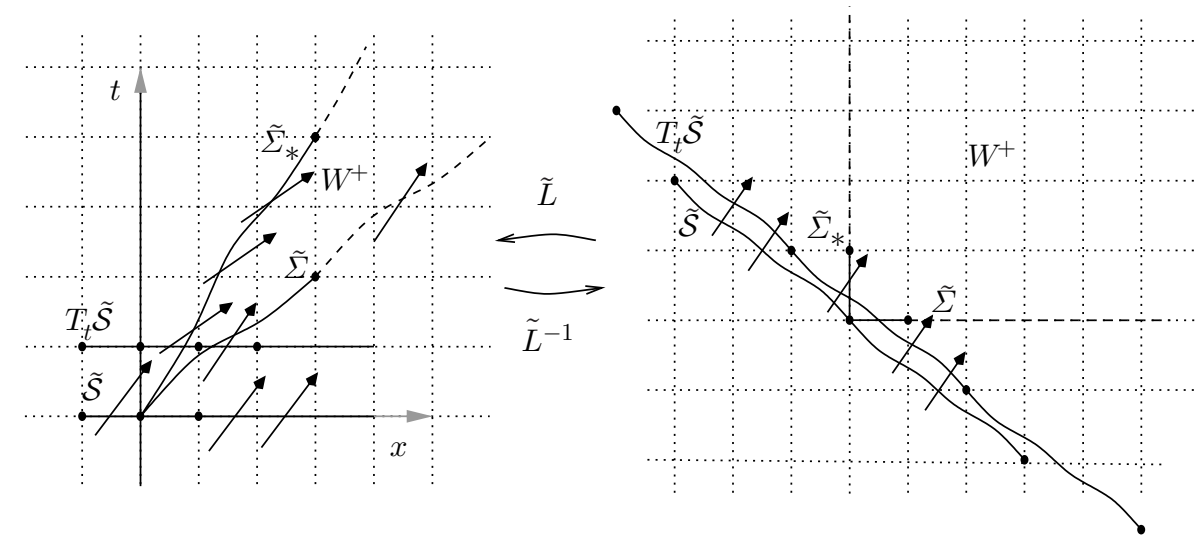

Figure 4.1. The Straightening Conjugacy. (Here, $\mathrm{p} / \mathrm{q}=4 / 5$ and $\mathrm{p}_{*} / \mathrm{q}_{*}=3 / 4$.) 


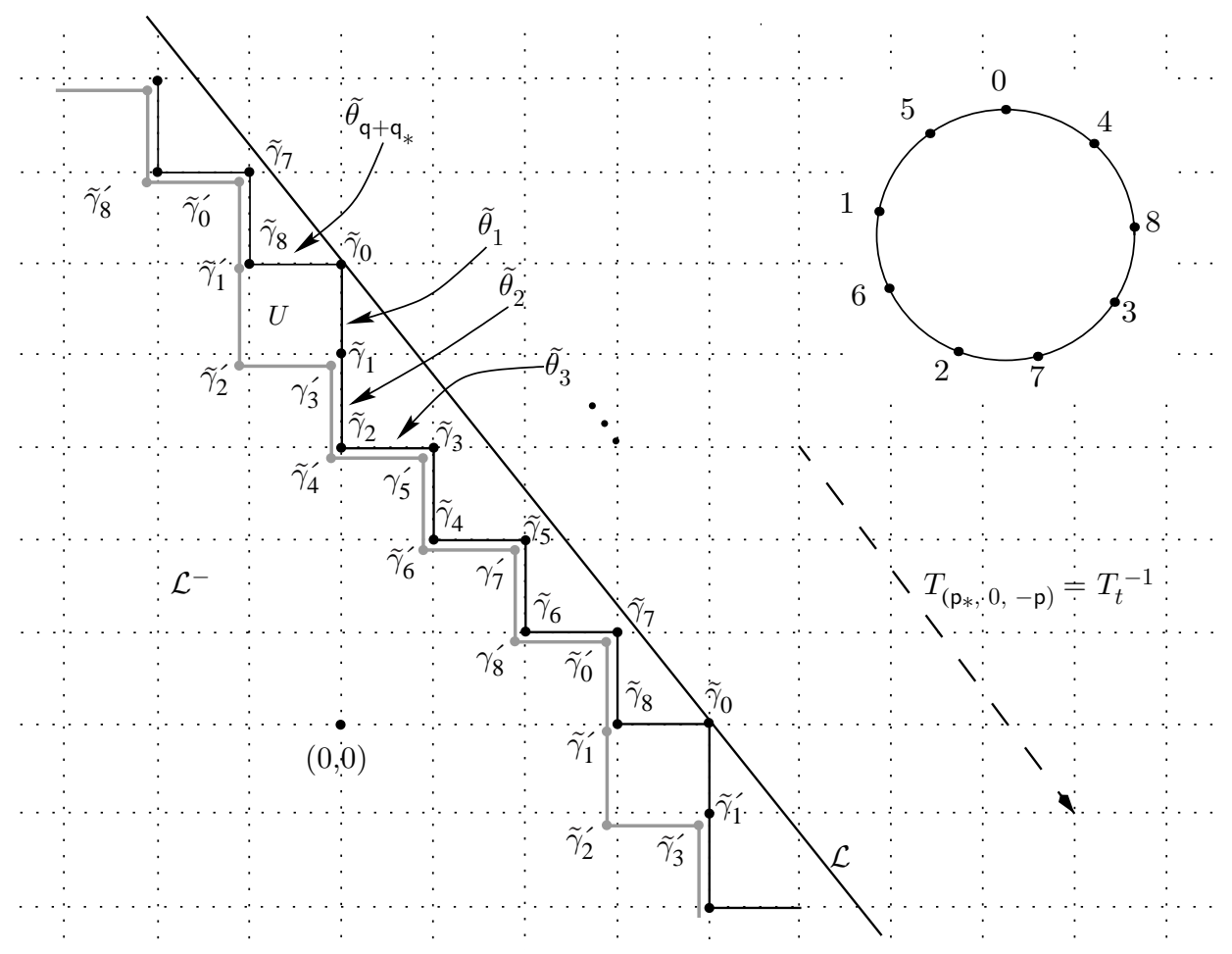

Figure 4.2. The Stepped Line. Here, $\mathrm{p} / \mathrm{q}=4 / 5<\alpha<3 / 4=$ $\mathrm{p}_{*} / \mathrm{q}_{*}$. The black stepped line is $\tilde{\theta}$ and the gray one is $T_{\mathrm{t}}{ }^{-1} \tilde{\theta}$. See that the lattice points on the circle $\tilde{\theta} / \mathbb{Z}\left(\mathbf{q}_{*},-\mathbf{q}\right)$ map under $T_{\mathrm{t}}^{-1}=T_{\left(\mathbf{p}_{*}, 0,-\mathrm{p}\right)}$ as follows: $\hat{\gamma}_{0} \rightarrow \hat{\gamma}_{7} \rightarrow \hat{\gamma}_{5} \rightarrow \hat{\gamma}_{3} \rightarrow \hat{\gamma}_{1} \rightarrow \hat{\gamma}_{8} \rightarrow$ $\hat{\gamma}_{6} \rightarrow \hat{\gamma}_{4} \rightarrow \hat{\gamma}_{2}$. The inset depicts the iterates of $T_{\mathrm{t}}{ }^{-i} \hat{\gamma}_{0}$ (labeled by $i)$; note the identical order to that under the circle rotation by $\alpha$.

Now, consider for a moment $\mathbb{R}^{2}$ (with coordinates $(x, t)$ ) and a straight line $\mathcal{L}$ through points $(0, \mathrm{q})$ and $\left(\mathrm{q}_{*}, 0\right)$ - see Figure 4.2 Let $\mathcal{L}^{-}$be the open halfspace bounded by $\mathcal{L}$ that contains $(0,0)$. Take the union of all the closed lattice squares (i.e. $\mathbb{Z}^{2}$ translates of $[0,1]^{2}$ ) with interior entirely contained in $\mathcal{L}^{-}$:

$$
\tilde{H}^{-}:=\bigcup\left\{Q: Q \text { is a lattice square with } \operatorname{int}(Q) \subset \mathcal{L}^{-}\right\} .
$$

Define $\tilde{\theta}$ as the boundary of $\tilde{H}^{-}$,

$$
\tilde{\theta}:=\partial \tilde{H}^{-},
$$

and observe that its projection $\hat{\theta}$ to the cylinder $\mathbb{R}^{2} / T_{\left(\mathbf{q}_{*},-\mathbf{q}\right)}$ is an essential loop because $\tilde{H}^{-}$is invariant under $T_{\left(\mathbf{q}_{*},-\mathbf{q}\right)}$. Finally, define

$$
\tilde{\Theta}:=\{(x, y, t):(x, t) \in \tilde{\theta}\} \cong \tilde{\theta} \times \mathbb{R} \subset \mathbb{R}^{3}
$$

and

$$
\hat{\Theta}:=\pi_{\mathbb{R}^{3} / \Lambda}(\tilde{\Theta}) \subset \mathbb{R}^{3} / \Lambda
$$


where $\pi_{\mathbb{R}^{3} / \Lambda}: \mathbb{R}^{3} \rightarrow \mathbb{R}^{3} / \Lambda$ is the natural projection. We set out to show that $\hat{\Theta}$ constitutes a cross-section to $\hat{\phi}$ and the induced return map (conjugated to $\hat{f}$ ) has a natural translation loop.

One easily observes that $\tilde{\theta}$ is a staircase-like broken line: as we traverse $\tilde{\theta}$ from $(0, \mathbf{q})$ to $\left(\mathbf{q}_{*}, 0\right)$, the line can only step down vertically or move to the right horizontally (i.e. either $x$ or $-t$ is increasing); in the process, we encounter $\mathrm{q}+\mathrm{q}_{*}$ segments, denoted by $\tilde{\theta}_{1}, \ldots, \tilde{\theta}_{\mathbf{q}+\mathbf{q}_{*}}$, with endpoints at the lattice points $\tilde{\gamma}_{i}:=\tilde{\theta}_{i} \cap \tilde{\theta}_{i+1}$, $i=0, \ldots, \mathbf{q}+\mathrm{q}_{*}-1$. Consult Figure 4.2 In $\mathbb{R}^{2} / \mathbb{Z}\left(\mathbf{q}_{*},-\mathbf{q}\right)$, we have the corresponding segments $\hat{\theta}_{1}, \ldots, \hat{\theta}_{\mathbf{q}+\mathbf{q}_{*}}$ covering the loop $\hat{\theta}$ and overlapping only at the endpoints $\hat{\gamma}_{0}, \ldots, \hat{\gamma}_{\mathbf{q}+\mathbf{q}_{*}-1}$. Therefore, by setting

$$
\hat{\Theta}_{i}:=\pi_{\mathbb{R}^{3} / \Lambda}\left(\tilde{\Theta}_{i}\right) \cong \hat{\theta}_{i} \times \mathbb{T},
$$

we get a covering of $\hat{\Theta}$ by $q+q_{*}$ annuli, $\hat{\Theta}_{1}, \ldots, \hat{\Theta}_{q+q_{*}}$, with pairwise disjoint interiors. Let $\hat{\Gamma}_{i}$ 's be the boundary circles of these annuli:

$$
\hat{\Gamma}_{i}:=\hat{\Theta}_{i} \cap \hat{\Theta}_{i+1} \cong \hat{\gamma}_{i} \times \mathbb{T},
$$

where $i=0, \ldots, \mathrm{q}+\mathrm{q}_{*}-1$ and indexing is modulo $\mathrm{q}+\mathrm{q}_{*} \cdot\left(\hat{\Gamma}_{0}\right.$ will be the sought after translation loop.)

Fact 4.1. Recall the "time translation" $T_{\mathrm{t}}:=T_{\left(-\mathrm{p}_{*}, 0, \mathrm{p}\right)}$.

(i) $\hat{\Theta}$ is compact and every flow line meets $\hat{\Theta}$ exactly once;

(ii) the holonomy $\hat{\phi}_{\hat{\Theta}}:=\left.\hat{\phi}_{\hat{\mathcal{S}}}\right|_{\hat{\Theta}}: \hat{\Theta} \rightarrow \hat{\mathcal{S}}$ is a homeomorphism;

(iii) $\left(\bigcup_{i=0}^{\mathrm{q}+\mathrm{q}_{*}-1} \hat{\Gamma}_{i}\right) \backslash T_{\mathrm{t}}^{-1}\left(\bigcup_{i=0}^{\mathrm{q}+\mathrm{q}_{*}-1} \hat{\Gamma}_{i}\right)=\hat{\Gamma}_{0}$;

(iv) $\bigcup_{i=0}^{\mathrm{q}+\mathrm{q}_{*}-1} \hat{\Gamma}_{i}=\left\{\hat{\Gamma}_{0}, T_{\mathrm{t}}^{-1}\left(\hat{\Gamma}_{0}\right), \ldots, T_{\mathrm{t}}^{-\left(\mathrm{q}+\mathrm{q}_{*}-1\right)}\left(\hat{\Gamma}_{0}\right)\right\}$.

Proof. (i) Since $\hat{\phi}$ is the lift of $\phi$ to the suspension covering, $\lim _{t \rightarrow \pm \infty} \hat{\phi}^{t}(p)=$ $\pm \infty$ where $\pm \infty$ denotes the two ideal boundary components of the cylinder $\mathbb{R}^{3} / \Lambda$. Because $\hat{\theta}$ separates $\mathbb{R}^{2} / T_{\left(\mathbf{q}_{*},-\mathbf{q}\right)}, \hat{\Theta}$ separates $\mathbb{R}^{3} / \Lambda$ into two connected components, which we denote $\hat{\Theta}^{-}$and $\hat{\Theta}^{+}$so that $-\infty \in \hat{\Theta}^{-}$and $+\infty \in \hat{\Theta}^{+}$. Hence, every flow line of $\hat{\phi}$ must intersect $\hat{\Theta}$. We have to argue that this intersection is unique. It suffices to show that $\hat{\Theta}$ is topologically transversal ${ }^{4}$ to the flow, i.e. if $p \in \hat{\Theta}$ then there is $\epsilon>0$ so that $\hat{\phi}^{[-\epsilon, 0)}(p) \subset \hat{\Theta}^{-}$and $\hat{\phi}^{(0, \epsilon]}(p) \subset \hat{\Theta}^{+}$. This, in turn, easily follows from the staircase-like shape of $\hat{\Theta}$, the flow's transversality to $\Sigma$ and $\Sigma_{*}$, and the forward invariance of the wedge $W^{+}$.

(ii) Both $\hat{\mathcal{S}}$ and $\hat{\Theta}$ intersects every flow line exactly once, so the continuous map $\left.\hat{\phi}_{\hat{\mathcal{S}}}\right|_{\hat{\Theta}}: \hat{\Theta} \rightarrow \hat{\mathcal{S}}$ is onto and one-to-one, and thus it is a homeomorphism by virtue of the compactness.

To show assertions (iii) and (iv) we establish the corresponding statements about $\hat{\theta}$ and the lattice points $\hat{\gamma}_{i}:=\hat{\theta}_{i} \cap \hat{\theta}_{i+1}$ under the action of $T_{\left(-\mathrm{p}_{*}, \mathrm{p}\right)}{ }^{-1}$ - see Figure 4.2.

(iii) Observe that $T_{\left(-\mathrm{p}_{*}, \mathrm{p}\right)}{ }^{-1}(\hat{\theta})$ sits in $H^{-}($below $\hat{\theta})$ because the definition of $\hat{\theta}$ is $T_{\left(-\mathrm{p}_{*}, \mathrm{p}\right)}$-equivariant and $T_{\left(-\mathrm{p}_{*}, 0, \mathrm{p}\right)}{ }^{-1}(\mathcal{L}) \subset \mathcal{L}^{-}$. Let $U$ then be the bounded region trapped between $\hat{\theta}$ and $T_{\left(-\mathrm{p}_{*}, \mathrm{p}\right)}{ }^{-1}(\hat{\theta})$. We claim that $U$ has unit area. Indeed, since $\mathrm{pq}_{*}-\mathrm{p}_{*} \mathrm{q}=1$, the area swept in the parallel translation of the segment $\left[(0, q),\left(q_{*}, 0\right)\right]$ by the vector $\left(-p_{*}, p\right)$ is one; and it coincides with the area of $U$

\footnotetext{
${ }^{4}$ Although, appropriately defined, smooth transversality also holds.
} 
because (by vanishing of divergence) the flux of the constant vector field $\left(-p_{*}, p\right)$ is the same through $\hat{\theta}$ and $\left[(0, \mathbf{q}),\left(\mathbf{q}_{*}, 0\right)\right]$ (or any of their translates). Hence, $U$ is a single lattice square. Therefore, all lattice points of $\hat{\theta}$ except for one - the upper right corner of $U$ in Figure 4.2 - belong to $T_{\left(-\mathrm{p}_{*}, \mathrm{p}\right)}{ }^{-1}(\hat{\theta})$. That the omitted lattice point is $\hat{\gamma}_{0}$, follows from $T_{\left(-\mathrm{p}_{*}, \mathrm{p}\right)} \hat{\gamma}_{0} \in \mathcal{L}^{+}$, which assures that $T_{\left(-\mathrm{p}_{*}, \mathrm{p}\right)} \hat{\gamma}_{0} \notin \hat{\theta}$.

(iv) Let $\delta: \mathbb{R}^{2} / T_{\left(\mathrm{q}_{*},-\mathrm{q}\right)} \rightarrow \mathbb{R}$ be the quotient of the orthogonal projection $\mathbb{R}^{2} \rightarrow \mathbb{R}$ along $\mathcal{L}$. It is easy to see that $\hat{\gamma}_{i}$ 's are exactly the points $v \in \mathbb{Z}^{2} / T_{\left(\mathbf{q}_{*},-\mathbf{q}\right)} \cap \operatorname{cl}\left(\mathcal{L}^{-}\right)$ with $\operatorname{dist}(v, \mathcal{L})<\sqrt{2} ;$ equivalently, $\left\{\hat{\gamma}_{i}\right\}_{i=0}^{\mathbf{q}+\mathbf{q}_{*}-1}=\left\{v \in \mathbb{Z}^{2} / T_{\left(\mathbf{q}_{*},-\mathbf{q}\right)}: \delta(v) \in\right.$ $(-\sqrt{2}, 0]\}$. Also, $T_{\left(-\mathrm{p}_{*}, \mathrm{p}\right)}$ pushes $\mathcal{L}$ to the right: there is $a>0$ such that $\delta\left(T_{\left(-\mathrm{p}_{*}, \mathrm{p}\right)}(p)\right)$ $=\delta(p)+a$ for all $p \in \mathbb{R}^{2} / T_{\left(\mathbf{q}_{*},-\mathbf{q}\right)}$. In view of (iii), there is a well defined $\lambda:\left\{1, \ldots, \mathbf{q}+\mathbf{q}_{*}-1\right\} \rightarrow\left\{0, \ldots, \mathbf{q}+\mathbf{q}_{*}-1\right\}$ such that $\lambda(i)=j$ iff $T_{\left(-\mathbf{p}_{*}, \mathbf{p}\right)}\left(\hat{\gamma}_{i}\right)=\hat{\gamma}_{j}$. We have to show that $\left\{0, \ldots, \mathbf{q}+\mathbf{q}_{*}-1\right\}=\left\{i_{0}, \lambda\left(i_{0}\right), \ldots, \lambda^{\mathbf{q}+\mathbf{q}_{*}-1}\left(i_{0}\right)\right\}$ for some $i_{0}$ with $\lambda^{\mathbf{q}+\mathbf{q}_{*}-1}\left(i_{0}\right)=0$. Observe that because $a>0, \lambda$ has no cycles and, for any $0 \leq i \leq \mathrm{q}+\mathrm{q}_{*}-1$, there is a minimal $k=k(i) \geq 0$ such that $\lambda^{k}(i)=0$. Clearly, $k(i) \leq \mathrm{q}+\mathrm{q}_{*}-1$ as $i, \lambda(i), \ldots, \lambda^{k(i)}(i)$ must all be distinct (for lack of cycles). Moreover, if $k(i)=k(j)$ then $i=j$ because $\lambda$ is one-to-one. Thus $k:\left\{0, \ldots, \mathrm{q}+\mathrm{q}_{*}-1\right\} \rightarrow\left\{0, \ldots, \mathrm{q}+\mathrm{q}_{*}-1\right\}$ is one-to-one and therefore onto. The $i_{0}:=k^{-1}\left(\mathbf{q}+\mathbf{q}_{*}-1\right)$ has the desired property.

Conclusion of the proof of Theorem 4.7. (i) and (ii) of Fact 4.1 secure the following commuting diagram

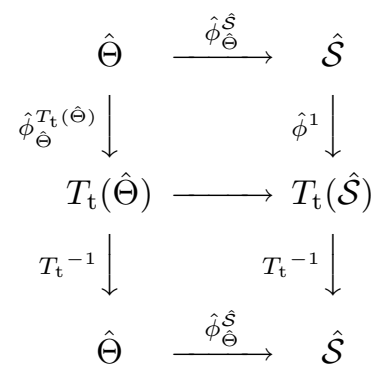

where $\hat{\phi}_{\hat{\Theta}}, \hat{\phi}_{\hat{\Theta}}^{\hat{S}}$, and the unlabeled arrow are the natural holonomies between crosssections. By (iii) and (iv) of Fact 4.1, we have that $\hat{\phi}_{\hat{\Theta}}^{T_{\mathrm{t}}(\hat{\Theta})}$ is the identity on the set $\left\{\hat{\Gamma}_{0}, \ldots, T_{\mathrm{t}}^{-\mathrm{q}-\mathrm{q}_{*}+2}\left(\hat{\Gamma}_{0}\right)\right\}$. If we recall (4.7) to the effect that $T_{\mathrm{t}}{ }^{-1} \circ \hat{\phi}^{1}=\hat{f}$, we deduce from (4.8) that the following diagram commutes

$$
\begin{array}{rr}
\left\{\hat{\Gamma}_{0}, T_{\mathrm{t}}^{-1}\left(\hat{\Gamma}_{0}\right), \ldots, T_{\mathrm{t}}^{-\mathbf{q}-\mathbf{q}_{*}+2}\left(\hat{\Gamma}_{0}\right)\right\} & \longrightarrow \hat{\mathcal{S}} \\
T_{\mathrm{t}}{ }^{-1} \downarrow & \hat{f} \downarrow \\
\left\{T_{\mathrm{t}}^{-1}\left(\hat{\Gamma}_{0}\right), \ldots, T_{\mathrm{t}}^{-\mathrm{q}-\mathbf{q}_{*}+1}\left(\hat{\Gamma}_{0}\right)\right\} & \longrightarrow \hat{\mathcal{S}}
\end{array}
$$

It is now easy to see that $\hat{\gamma}:=\hat{\phi}_{\hat{\Theta}}\left(\hat{\Gamma}_{0}\right)$ is the sought after translation loop with the required ordering of its iterates. Indeed, the homeomorphisms $h$ asserted in the theorem can be constructed as follows. Let $h_{f}: \hat{\Theta} \rightarrow \mathbb{T}^{2}$ be the composition of $\hat{\phi}_{\hat{\Theta}}^{\hat{\mathcal{S}}}$ with the identification between $\hat{\mathcal{S}}$ and $\mathbb{T}^{2}$ (conjugating $\hat{f}$ with $f$ ). Let also $h_{\alpha}$ : $\hat{\Theta} \rightarrow \mathbb{T}^{2}$ be the analogous homeomorphisms when $f$ is replaced by the translation $T_{\alpha}:(x, y) \mapsto(x+\alpha, y) \quad\left(\bmod \mathbb{Z}^{2}\right)$ (which obviously satisfies all the hypotheses of the theorem). That $h=h_{\alpha} \circ h_{f}^{-1}$ is as required follows readily by putting together 
(4.8) and (4.9) with their counterparts for $T_{\alpha}$. This completes the proof of Theorem 4.7.

\section{RenORMALization}

This section is a digression and the readers interested only in the proof of Theorem 1.1 should move on to Section 6 after reading Definition 5.2 below.

Definition 5.2. Given $A=\left(\begin{array}{cc}\mathrm{p} & \mathrm{p}_{*} \\ \mathrm{q} & \mathrm{q}_{*}\end{array}\right) \in \mathbf{S L}_{2}(\mathbb{Z})$ with $\mathrm{q}, \mathrm{q}_{*}>0$, a map $f \in \operatorname{Diff}_{0}\left(\mathbb{T}^{2}\right)$ is A-renormalizable iff there is a simple $(0,1)$-loop $\gamma$ such that $\left(f^{i}(\gamma)\right)_{j=0}^{\mathbf{q}+\mathbf{q}_{*}-1}$ are disjoint and ordered as $(j \alpha(\bmod 1))_{j=0}^{\mathbf{q}+\mathrm{q}_{*}-1}$ (in the sense of Theorem 4.7), where $\alpha$ is any number between $\mathrm{p} / \mathrm{q}$ and $\mathrm{p}_{*} / \mathrm{q}_{*}$.

In what follows, we attempt to shed some light on the definition by linking renormalization with flow equivalence. As we have already mentioned, a more complete discussion is relegated to [23].

Proposition 5.1. Suppose that $f \in \operatorname{Diff}_{0}\left(\mathbb{T}^{2}\right)$ is A-renormalizable, $J$ is the annulus bounded by $\gamma$ and $f^{\mathrm{q}}(\gamma)$ and $J / \sim$ is the torus obtained by identifying the boundary curves of $J$ via $f^{q}$. Then the suspension flow of $f$ has a well defined crosssection $\Sigma$ in the cohomology class $(-\mathrm{q}, 0, \mathrm{p})$ (represented by $\operatorname{lin}\{(p, 0, q),(0,1,0)\}$ $\left.\left(\bmod \mathbb{Z}^{2}\right)\right)$; and the map $\mathcal{R}_{J}(f): J / \sim \rightarrow J / \sim$ induced by the first return to $J$ under $f$ is conjugated to the Poincaré return map $\phi_{\Sigma}: \Sigma \rightarrow \Sigma$ for the flow.

The analogous proposition holds for the annulus $J_{*}$ bounded by $\gamma$ and $f^{\mathbf{q}_{*}}(\gamma)$.

Corollary 5.2. $f \in \operatorname{Diff}_{0}\left(\mathbb{T}^{2}\right)$ is A-renormalizable if and only if $\rho(\tilde{f}) \subset\left(\mathrm{p}_{*} / \mathrm{q}_{*}\right.$, $\mathrm{p} / \mathrm{q}) \times \mathbb{R}$ for some lift $\tilde{f}$ of $f$.

Proof of Corollary 5.2. If $f$ is $A$-renormalizable, then by the proposition we have well defined cross-sections in the cohomology classes of $\operatorname{lin}\{(\mathrm{p}, 0, \mathrm{q}),(0,1,0)\}$ $\left(\bmod \mathbb{Z}^{2}\right)$ and $\operatorname{lin}\left\{\left(\mathrm{p}_{*}, 0, \mathbf{q}_{*}\right),(0,1,0)\right\}\left(\bmod \mathbb{Z}^{2}\right)$, which implies the condition on the rotation set by (2.4). The opposite implication is the content of Theorem 4.7

Sketch of Proof of Proposition [5.1] Let us first assume that $\gamma$ is obtained from the intersection of a clean pair of cross-sections $\Sigma$ and $\Sigma_{*}$. It suffices to show that the first return map under $f, f_{J \cup J_{*}}: J \cup J_{*} \rightarrow J \cup J_{*}$, is conjugated to the flow's first return map to $\Sigma \cup \Sigma_{*}, \phi_{\Sigma \cup \Sigma_{*}}: \Sigma \cup \Sigma_{*} \rightarrow \Sigma \cup \Sigma_{*}$. Indeed, $\phi_{\Sigma}: \Sigma \rightarrow \Sigma$ is simply the first return map to $\Sigma$ under $\phi_{\Sigma \cup \Sigma_{*}}$. We shall use the notations of the proof of Theorem 4.7 as found in the previous section. In particular, we have the holonomy $\hat{\phi} \hat{\hat{\mathcal{S}}}: \hat{\Theta} \rightarrow \hat{\mathcal{S}}$ that served to define $\gamma:=\pi_{\mathbb{T}^{3}} \circ \hat{\phi}_{\hat{\Theta}}^{\hat{\mathcal{S}}}\left(\hat{\Gamma}_{0}\right)$, and we have the stepped torus $\hat{\Theta}=\bigcup_{i=1}^{\mathbf{q}+\mathbf{q}_{*}} \hat{\Theta}_{i}$ where each $\hat{\Theta}_{i} \subset \mathbb{R}^{3} / \Lambda$ is a lift of one of the two (straightened) clean cross-sections $\Sigma$ and $\Sigma_{*}$ in $\mathbb{T}^{3}$. Specifically, $\hat{\Theta}_{1}$ and $\hat{\Theta}_{\mathbf{q}+\mathbf{q}_{*}}$ are lifts of $\Sigma$ and $\Sigma_{*}$, respectively - see Figure 4.2. Together with $T_{\mathrm{t}}{ }^{-\mathrm{q}}\left(\hat{\Theta}_{1}\right)$ and $T_{\mathrm{t}}{ }^{-\mathrm{q}_{*}}\left(\hat{\Theta}_{\mathrm{q}+\mathrm{q}_{*}-1}\right)$, they form the boundary of the region $U \times \mathbb{T} \subset \mathbb{R}^{3} / \Lambda$, where $U$ is the lattice square with its max vertex at $\hat{\gamma}_{0}$ (as defined in the proof of (iii) of Fact 4.1). A slice through $U \times \mathbb{T}$ is depicted in Figure 5.1. The flow enters $U \times \mathbb{T} \subset \mathbb{R}^{3} / \Lambda$ via $T_{\mathrm{t}}{ }^{-\mathrm{q}}\left(\hat{\Theta}_{1}\right) \cup T_{\mathrm{t}}{ }^{-\mathrm{q}_{*}}\left(\hat{\Theta}_{\mathrm{q}+\mathrm{q}_{*}}\right)$ only to leave via $\hat{\Theta}_{1} \cup \hat{\Theta}_{\mathrm{q}+\mathrm{q}_{*}}$; let $\psi: T_{\mathrm{t}}{ }^{-\mathrm{q}}\left(\hat{\Theta}_{1}\right) \cup T_{\mathrm{t}}{ }^{-\mathrm{q}_{*}}\left(\hat{\Theta}_{\mathrm{q}+\mathrm{q}_{*}}\right) \rightarrow \hat{\Theta}_{1} \cup \hat{\Theta}_{\mathrm{q}+\mathrm{q}_{*}}$ be the associated holonomy. 


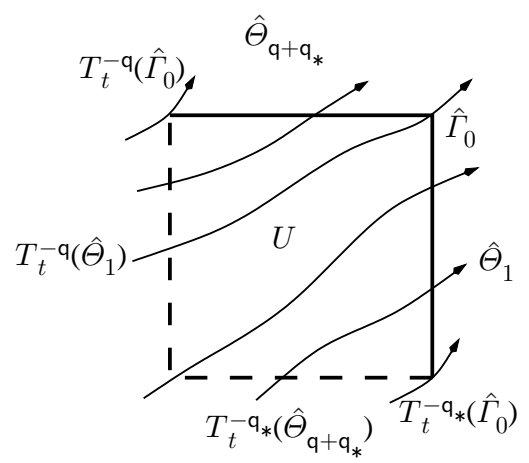

Figure 5.1. The Return Map.

Now, from diagram (4.8), $f$ is conjugated to the map on $\hat{\Theta}$ given by

$$
f^{\prime}:=T_{\mathrm{t}}{ }^{-1} \circ \hat{\phi}_{\hat{\Theta}}^{T_{\mathrm{t}} \hat{\Theta}}=\hat{\phi}_{T_{\mathrm{t}}-1}^{\hat{\Theta}} \hat{\Theta}^{-} \circ T_{\mathrm{t}}^{-1}
$$

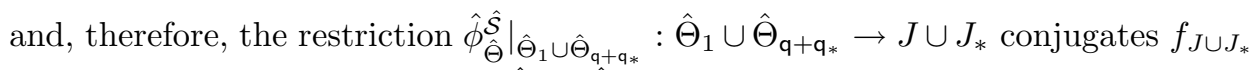
to $f_{\hat{\Theta}_{1} \cup \hat{\Theta}_{\mathrm{q}+\mathrm{q}_{*}}}$ (the return map to $\hat{\Theta}_{1} \cup \hat{\Theta}_{\mathrm{q}+\mathrm{q}_{*}}$ under $f^{\prime}$ ).

At the same time $f^{\prime}=T_{\mathrm{t}}^{-1}$ on most of $\hat{\Theta}$ (see (iii) of Fact 4.1), and from the action of $T_{\mathrm{t}}^{-1}$ on $\hat{\Theta}$ (see (iv) of Fact 4.1 ), one easily computes

$$
f_{\hat{\Theta}_{1} \cup \hat{\Theta}_{\mathrm{q}+\mathrm{q}_{*}}}^{\prime}(p)= \begin{cases}\hat{\phi}_{T_{\mathrm{t}}-1}^{\hat{\Theta}^{-}} \circ T_{\mathrm{t}}^{-\mathrm{q}}(p)=\psi \circ T_{\mathrm{t}}^{-\mathrm{q}}(p) & \text { if } p \in \hat{\Theta}_{1} ; \\ \hat{\phi}_{T_{\mathrm{t}}-1}^{\hat{\Theta}} \circ T_{\mathrm{t}}^{-\mathrm{q}_{*}}(p)=\psi \circ T_{\mathrm{t}}^{-\mathrm{q}_{*}}(p) & \text { if } p \in \hat{\Theta}_{\mathrm{q}+\mathrm{q}_{*}} .\end{cases}
$$

Descending to $\mathbb{T}^{3}$, we see from the above formula that $f_{\hat{\Theta}_{1} \cup \hat{\Theta}_{\mathrm{q}+q_{*}}}^{\prime}$ factors to the return map $\phi_{\Sigma \cup \Sigma_{*}}$. This shows that $\phi_{\Sigma \cup \Sigma_{*}}$ is conjugated to $f_{J \cup J_{*}}-$ as we needed to prove.

To finish the proof, we have to argue that if $f \in \operatorname{Diff}_{0}\left(\mathbb{T}^{2}\right)$ is $A$-renormalizable, then the translation loop $\gamma$ arises from the intersection of a clean pair of crosssections $\Sigma$ and $\Sigma_{*}$ to the suspension flow (in the appropriate cohomology classes).

Consider now the 2-torus $\hat{\Theta}=\bigcup_{i=1}^{\mathbf{q}+\mathbf{q}_{*}} \hat{\Theta}_{i}$. Because the ordering of $\left(f^{j}(\gamma)\right)_{j=0}^{\mathbf{q}+\mathbf{q}_{*}-1}$ in $\mathbb{T}^{2}$ is the same as that of $\left(T_{\mathrm{t}}^{-j}\left(\hat{\Gamma}_{0}\right)\right)_{j=0}^{\mathbf{q}+\mathbf{q}_{*}-1}$ in $\hat{\Theta}$, one easily constructs a homeomorphism $h: \mathbb{T}^{2} \rightarrow \hat{\Theta}$ that maps $f^{j}(\gamma)$ to $T_{\mathrm{t}}^{-j}\left(\hat{\Gamma}_{0}\right)$ for $0 \leq j \leq \mathrm{q}+$ $\mathrm{q}_{*}-1$ and such that $f^{\prime}:=h \circ f \circ h^{-1}$ satisfies $\left.f^{\prime}\right|_{T_{\mathrm{t}}-j}\left(\hat{\Theta}_{1}\right)=T_{\mathrm{t}}^{-1}$ for $j=$ $0, \ldots, \mathrm{q}-2$ and $\left.f^{\prime}\right|_{T_{\mathrm{t}}-j}\left(\hat{\Theta}_{\mathrm{q}+\mathrm{q}_{*}}\right)=T_{\mathrm{t}}^{-1}$ for $j=0, \ldots, \mathbf{q}_{*}-2$. On $T_{\mathrm{t}}^{-\mathrm{q}+1}\left(\hat{\Theta}_{1}\right)$

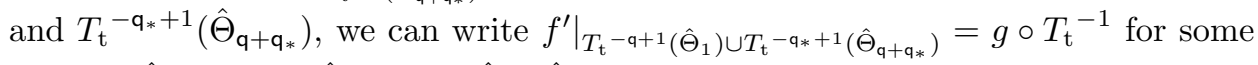
$g: T_{\mathrm{t}}^{-\mathrm{q}}\left(\hat{\Theta}_{1}\right) \cup T_{\mathrm{t}}^{-\mathrm{q}_{*}}\left(\hat{\Theta}_{\mathrm{q}+\mathrm{q}_{*}}\right) \rightarrow \hat{\Theta}_{1} \cup \hat{\Theta}_{\mathrm{q}+\mathrm{q}_{*}}$. Now, a routine construction yields a flow supported in $U \times \mathbb{T}$ that enters via $T_{\mathrm{t}}^{-\mathrm{q}}\left(\hat{\Theta}_{1}\right) \cup T_{\mathrm{t}}^{-\mathrm{q}_{*}}\left(\hat{\Theta}_{\mathbf{q}+\mathrm{q}_{*}}\right)$, leaves via $\hat{\Theta}_{1} \cup \hat{\Theta}_{\mathbf{q}+\mathrm{q}_{*}}$, and realizes $g$ as its holonomy. This flow factors down to a certain flow $\phi$ on $\mathbb{T}^{3}$ (since $U \times \mathbb{T}$ is a fundamental domain). By construction, $\Sigma=\operatorname{lin}\{(1,0,0),(0,1,0)\}$ $\left(\bmod \mathbb{Z}^{3}\right)$ and $\Sigma=\operatorname{lin}\{(0,0,1),(0,1,0)\}\left(\bmod \mathbb{Z}^{3}\right)$ are cross-sections to $\phi ;$ and $\phi$ is (conjugated to) the suspension flow of $f^{\prime}$ or, equivalently, the suspension flow of $f$. 
It is also natural to consider $\mathcal{R}_{J \cup J_{*}}(f):\left(J \cup J_{*}\right) / \sim \rightarrow\left(J \cup J_{*}\right) / \sim$ induced by the return map to $J \cup J_{*}$ with $\sim$ being again the natural identification of the boundaries (which makes $\left(J \cup J_{*}\right) / \sim$ a torus).

Corollary 5.3. The conjugacy classes of $\mathcal{R}_{J}(f): J / \sim \rightarrow J / \sim, \mathcal{R}_{J_{*}}(f): J_{*} / \sim \rightarrow$ $J_{*} / \sim$, and $\mathcal{R}_{J \cup J_{*}}(f):\left(J \cup J_{*}\right) / \sim \rightarrow\left(J \cup J_{*}\right) / \sim$ are independent of the choice of $\gamma$. Moreover, they are flow equivalent to $f$.

Sketch of Proof of Corollary 5.3. Proposition 5.1 contains the assertion about $\mathcal{R}_{J}(f)$. As to $\mathcal{R}_{J \cup J_{*}}(f)$, the two cross sections $\Sigma$ and $\Sigma_{*}$ constructed in the proof of the proposition can be cut along $\Sigma \cap \Sigma_{*}$, perturbed, and glued back together to form a cross-section in the cohomology class of $\operatorname{lin}\left\{\left(\mathrm{p}-\mathrm{p}_{*}, 0, \mathrm{q}-\mathrm{q}_{*}\right),(0,1,0)\right\}$ $\left(\bmod \mathbb{Z}^{3}\right)$. $\quad \mathcal{R}_{J \cup J_{*}}(f)$ is naturally conjugated to the corresponding return map (much like $\mathcal{R}_{J}(f)$ ).

Any map of the form $\eta \circ \mathcal{R}_{J}(f) \circ \eta^{-1}: \mathbb{T}^{2} \rightarrow \mathbb{T}^{2}$ (where $\eta: \mathcal{R}_{J}(f) \rightarrow \mathbb{T}^{2}$ is a homeomorphisms) is called a $p / q$-renormalization of $f$. Likewise, any map of the form $\eta \circ \mathcal{R}_{J \cup J_{*}}(f) \circ \eta^{-1}: \mathbb{T}^{2} \rightarrow \mathbb{T}^{2}$ (with $\eta: \mathcal{R}_{J \cup J_{*}}(f) \rightarrow \mathbb{T}^{2}$ ) is called an A-renormalization of $f$.

Before leaving this section, we note that doubling the annulus to a torus and applying Theorem 4.7 yields the following result.

Corollary 5.4. Suppose that $f: \mathbb{T} \times[0,1] \rightarrow \mathbb{T} \times[0,1]$ is an orientation preserving diffeomorphism isotopic to the identity and $\tilde{f}: \mathbb{R} \times[0,1] \rightarrow \mathbb{R} \times[0,1]$ is its lift. If $\tilde{f}$ has a well defined and irrational rotation number $\alpha$ (i.e. $\lim _{n \rightarrow \infty} \frac{1}{n} \operatorname{pr}_{\mathbb{R}}\left(\tilde{f}^{n}(p)-p\right)=$ $\alpha$ for all $p \in \mathbb{R} \times[0,1])$, then $f$ is infinitely renormalizable in the sense that, for every pair of Farey neighbors $\mathrm{p} / \mathrm{q}$ and $\mathrm{p}_{*} / \mathrm{q}_{*}$, there exists a simple curve $\gamma$ joining the two boundary components of $\mathbb{T} \times[0,1]$ such that $\left(f^{i}(\gamma)\right)_{j=0}^{\mathrm{q}+\mathrm{q}_{*}-1}$ are disjoint and ordered as $(j \alpha(\bmod 1))_{j=0}^{\mathrm{q}+\mathrm{q}_{*}-1}$.

There is a conjecture (dating back to G. Birkhoff) that if $f$ is an area preserving and sufficiently smooth annulus map satisfying the hypotheses of the corollary, then $f$ is actually conjugated to the rigid rotation for Diophantine $\alpha$ [14]. Corollary [5.4 may be a basis for the global renormalization approach to this question (cf. Question 3.2 in [14]).

\section{Rotation Estimates}

This section contains the analytical part of the proof of Theorem 1.1. By virtue of the the translation loop theorem (Theorem 4.7) we may assume that $f \in \mathbf{D i f f}_{0}\left(\mathbb{T}^{2}\right)$ is $A$-renormalizable for $A=\left(\begin{array}{ll}\mathrm{p} & \mathrm{p}_{*} \\ \mathrm{q} & \mathrm{q}_{*}\end{array}\right) \in \mathbf{S L}_{2}(\mathbb{Z})$ where $\mathrm{p} / \mathrm{q}$ and $\mathrm{p}_{*} / \mathrm{q}_{*}$ constitute a pair of Farey neighbors approximating $\alpha$. Theorem 1.1 follows by letting $\mathrm{p} / \mathrm{q}$ and $\mathrm{p}_{*} / \mathrm{q}_{*}$ converge to $\alpha$ and applying the following more precise result.

Theorem 6.8. There exists $C:[1, \infty) \times \mathbb{N} \rightarrow(0,+\infty)$ such that $\lim _{m \rightarrow \infty} C(K, m)=$ 0 for every $K>1$ and, if $f \in \operatorname{Diff}_{0}\left(\mathbb{T}^{2}\right)$ is $K$-quasiconformal and $A$-renormalizable with a lift $\tilde{f}$, then

$$
\operatorname{diam}(\rho(\tilde{f})) \leq C\left(K, \max \left\{\mathbf{q}, \mathbf{q}_{*}\right\}\right)
$$


The rest of this section is devoted to the proof of Theorem 6.8 In addition to $f \in \operatorname{Diff}_{0}\left(\mathbb{T}^{2}\right)$, we fix its lift $\tilde{f} \in \operatorname{Diff}_{0}\left(\mathbb{R}^{2}\right)$ and the translation loop $\gamma \subset \mathbb{T}^{2}$ as in Definition 5.2 For specificity, we shall assume that

$$
\mathrm{q}_{*}>\mathrm{q}
$$

Writing $\gamma_{j}:=f^{j}(\gamma)$, we take $J_{j}$ to be the closed annulus in $\mathbb{T}^{2}$ bounded by $\gamma_{j}$ and $\gamma_{j+\mathrm{q}}$; and $J_{* j}$ to be the closed annulus in $\mathbb{T}^{2}$ bounded by $\gamma_{j}$ and $\gamma_{j+\mathrm{q}_{*}}$ (cf. Figure 1.1). For $j \in \mathbb{Z}$, set

$$
U_{j}:=J_{j} \cup J_{* j}, \quad j \in \mathbb{Z} .
$$

Also, choose a lift $\tilde{U}_{0}$ of $U_{0}$ and set $\tilde{U}_{j}:=\tilde{f}^{j}\left(\tilde{U}_{0}\right)$.

The ordering of $(j \alpha)_{j=0}^{\mathbf{q}+\mathbf{q}_{*}-1}$ on $\mathbb{T}$ secures the following hypotheses for $k \in \mathbb{Z}$.

- Covering Property: $\left\{U_{k+j}\right\}_{j=0}^{\mathbf{q}_{*}-1}$ covers $\mathbb{T}^{2}: \bigcup_{j=0}^{\mathbf{q}_{*}-1} U_{k+j}=\mathbb{T}^{2}$;

- Overlap Property: $\left\{U_{k+j}\right\}_{j=0}^{\mathbf{q}_{*}-1}$ overlaps no more than twice: $U_{k+j}:=$ $J_{k+j} \cup J_{* k+j}$ and each family $\left\{J_{k+j}\right\}_{j=0}^{\mathrm{q}_{*}-1}$ and $\left\{J_{* k+j}\right\}_{j=0}^{\mathrm{q}_{*}-1}$ is essentially disjoint (i.e. the interiors are pairwise disjoint).

The sole role of Covering Property is to assure the following:

Fact 6.2. $\rho(\tilde{f})=\rho\left(\tilde{f}, U_{0}\right)$ (where $\rho\left(\tilde{f}, U_{0}\right)$ is defined as in 1.1) with the extra requirement that $\left.p \in U_{0}\right)$.

Proof. Only $\rho(\tilde{f}) \subset \rho\left(\tilde{f}, U_{0}\right)$ requires an argument. Consider $v \in \rho(\tilde{f})$, that is $v=\lim _{j \rightarrow \infty} \tilde{f}^{n_{j}}\left(\tilde{p}_{j}\right) / n_{j}$ for some $\tilde{p}_{j} \in \mathbb{R}^{2}$ and $n_{j} \rightarrow \infty$. By Covering Property, there are $k_{j}, 0 \leq k_{j} \leq \mathbf{q}_{*}-1$, such that $p_{j} \in U_{k_{j}}$; equivalently, $p_{j}^{\prime}:=f^{-k_{j}}\left(p_{j}\right) \in U_{0}$. Having fixed lifts $\tilde{p}_{j}^{\prime}$ of $p_{j}^{\prime}$, we estimate

$$
\left\|\tilde{f}^{n_{j}}\left(\tilde{p}_{j}^{\prime}\right)-\tilde{f}^{n_{j}}\left(\tilde{p}_{j}\right)\right\| \leq C:=\max _{y \in \mathbb{R}^{2}, 0 \leq k \leq \mathbf{q}_{*}-1}\left\|\tilde{f}^{k}(y)-y\right\|
$$

It follows that $v=\lim _{j \rightarrow \infty} \tilde{f}^{n_{j}}\left(\tilde{p}_{j}\right) / n_{j}=\lim _{j \rightarrow \infty} \tilde{f}^{n_{j}}\left(\tilde{p}_{j}^{\prime}\right) / n_{j} \in \rho\left(f, U_{0}\right)$.

Overlap Property is to be viewed in the context of the following variation on the superadditive property of the conformal modulus demonstrated in Section 7 . (Below, we assume the standard identification of $\mathbb{R}^{2}$ with the complex numbers $\mathbb{C}$ and consider $\mathbb{T}^{2}=\mathbb{R}^{2} / \mathbb{Z}^{2}$ as the conformal torus $\mathbb{T}^{2}=\mathbb{C} /(\mathbb{Z}+i \mathbb{Z})$.)

Lemma 6.1 (superadditivity). Suppose that $\left\{V_{j}\right\}_{j=1}^{N}$ is a finite family of embedded annuli in $\mathbb{T}^{2}$ each of homology type $(0,1)$, and suppose that $\left\{V_{j}\right\}_{j=1}^{N}$ has the $m$ overlap property defined as follows: there are $m$ essentially disjoint families $\left\{V_{j}^{l}\right\}_{j=1}^{N}$, $l=1, \ldots, m$, of $(0,1)$-annuli in $\mathbb{T}^{2}$ such that $\left\{V_{j}^{l}\right\}_{l=1}^{m}$ are also essentially disjoint and cover $V_{j}$ for each $j=1, \ldots, N$. Then

$$
\sum_{j=1}^{N} \bmod _{\mathbb{T}^{2}}\left(V_{j}\right) \leq m^{3}
$$

where $\bmod _{\mathbb{T}^{2}}\left(V_{j}\right) \leq 1$ stands for the modulus of the annulus $V_{j}$ in $\mathbb{T}^{2}$.

If we denote

$$
a_{j}:=\bmod _{\mathbb{T}^{2}}\left(U_{j}\right),
$$

then the lemma applied to $\left\{U_{k+j}\right\}_{j=0}^{\mathrm{q}_{*}-1}$ yields 
Fact 6.3. For $k \in \mathbb{Z}, a_{k} \leq 1$ and $\sum_{j=k}^{k+\mathrm{q}_{*}-1} a_{j} \leq 8$.

To proceed, we fix a (reference) point

$$
q \in f^{\mathrm{q}}(\gamma) \subset \partial U_{0}
$$

and its lift $\tilde{q} \in \mathbb{C}$. We also set, for $j \geq 0$,

$$
q_{j}:=f^{j}(q) \quad \text { and } \quad \tilde{q}_{j}:=\tilde{f}^{j}(\tilde{q}) .
$$

Our ultimate goal is to estimate the difference $\operatorname{Im}\left(\tilde{f}^{n}(\tilde{q})\right)-\operatorname{Im}\left(\tilde{f}^{n}(\tilde{p})\right)$ for any $\tilde{p}$ with $\pi(\tilde{p}) \in U_{0}$. The idea is to first perform the analogous comparison in the intrinsic uniformizing conformal coordinates on $U_{j}$ 's (cf. Claim 6.1 ahead). To this end, we denote by $B_{a}$ the cylinder of (modulus $a$ ) obtained as the quotient of the strip

$$
\tilde{B}_{a}:=\{z \in \mathbb{C}: 0 \leq \operatorname{Re}(z) \leq a\}
$$

by the $\mathbb{Z}$-action generated by $z \mapsto z+i$. For each $j \geq 0$, let $\psi_{j}: B_{a_{j}} \rightarrow U_{j}$ be a homeomorphism conformal on the interior of $B_{a_{j}}$; and select lifts $\tilde{\psi}_{j}: \tilde{B}_{a_{j}} \rightarrow \tilde{U}_{j}$, $\pi_{j} \circ \tilde{\psi}_{j}=\psi_{j} \circ \pi_{j}$, where $\pi_{j}: \tilde{B}_{a_{j}} \rightarrow B_{a_{j}}$ is the canonical projection. The dynamics induces $\tilde{f}_{j}: \tilde{B}_{a_{j}} \rightarrow \tilde{B}_{a_{j+1}}$ defined by the diagram

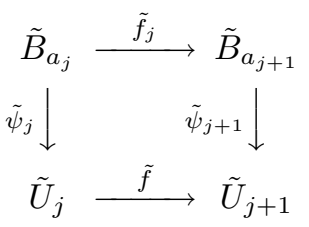

The restrictions of $\tilde{f}_{j}$ 's to the imaginary line line are

$$
\tilde{g}_{j}: \mathbb{R} \rightarrow \mathbb{R}, \quad g_{j}(y):=\tilde{f}_{j}(i y) / i, \quad j \geq 0 .
$$

Since $\psi_{j}$ are determined only up to rotation, we can impose a normalization

$$
\tilde{\psi}_{j}\left(\tilde{q}_{j}\right)=0
$$

so that

$$
\tilde{f}_{j}(0)=0, \quad \tilde{g}_{j}(0)=0, \quad j \geq 0 .
$$

We introduce now, for $K \geq 1$, a family $\mathcal{F}^{\mathbb{C}}{ }_{K}$ of maps $\phi$ satisfying the following conditions:

(i) $\phi: B_{a} \rightarrow B_{b}$ is a $K$-quasiconformal homeomorphism with $a, b<1$;

(ii) $\phi(z+i)=\phi(z)+i$ for all $z$;

(iii) $\phi(0)=0$.

The corresponding family of restrictions to the imaginary axis is

$$
\mathcal{F}^{\mathbb{R}}{ }_{K}:=\left\{\mu: \mathbb{R} \rightarrow \mathbb{R}: \exists \phi \in \mathcal{F}^{\mathbb{C}}{ }_{K} \quad \mu(y):=\phi(i y) / i \text { for all } y \in \mathbb{R}\right\} .
$$

We record the key property of $\tilde{f}_{j}$ 's.

Fact 6.4. Let $K:=K(f)$ be the quasiconformal distortion of $f$. The maps $\tilde{f}_{j}$ and $\tilde{f}_{j}^{-1}$ belong to $\mathcal{F}^{\mathbb{C}}{ }_{K}$; and thus $\tilde{g}_{j}$ and $\tilde{g}_{j}^{-1}$ belong to $\mathcal{F}^{\mathbb{R}}{ }_{K}$.

This is a consequence of the following variation on the classical result of Mori. 
Lemma 6.2 (Mori). $\mathcal{F}^{\mathbb{C}}{ }_{K}$ (and thus $\mathcal{F}^{\mathbb{R}}{ }_{K}$ ) is an equicontinuou 5 family (i.e. given $K>0$ and $\epsilon>0$, there is $\delta>0$ such that $|z-w| \leq \delta$ implies $|\phi(z)-\phi(w)| \leq \epsilon$ for any $\phi \in \mathcal{F}_{K}^{\mathbb{C}}$ ).

Define $G_{K}: \mathbb{R} \rightarrow \mathbb{R}$ as the upper envelope of $\mathcal{F}^{\mathbb{C}}{ }_{K}$ :

$$
G_{K}(y):=\sup \left\{\mu(y): \mu \in \mathcal{F}^{\mathbb{R}}{ }_{K}\right\} .
$$

The equicontinuity and monotonicity of functions in $\mathcal{F}^{\mathbb{R}}{ }_{K}$ and the fact that $\mu \in$ $\mathcal{F}^{\mathbb{R}}{ }_{K}$ implies $\mu^{-1} \in \mathcal{F}^{\mathbb{R}}{ }_{K}$ guarantee that $G_{K}$ is a continuous increasing function. Also, $G_{K}(y+k)=G_{K}(k)+y$ so that $G_{K}$ is a lift of an orientation preserving circle homeomorphism. Moreover, $G_{K}(y) \geq y$ for $y \in \mathbb{R}$ with $G_{K}(k)=0$ exactly for $k \in \mathbb{Z}$. In particular, the rotation number $\rho\left(G_{K}\right)=0$.

Fact 6.5. For $j \in \mathbb{Z}, x \in\left[0, a_{j}\right]$, and $y \in \mathbb{R}$,

$$
\operatorname{Im}\left(\tilde{f}_{j}(x+i y)\right) \leq G_{K}(y)+2 K a_{j+1} .
$$

Proof. Again, this depends on the following general bound demonstrated in the following section.

Lemma 6.3 (Shear Estimate). If $0<a, b<1$ and $\phi: B_{a} \rightarrow B_{b}$ is $K$-quasiconformal, then

$$
|\operatorname{Im}(\phi(x+i y))-\operatorname{Im}(\phi(0+i y))| \leq 2 K b, \quad 0 \leq x \leq a, y \in \mathbb{R} .
$$

Indeed, from the lemma and the definition of $G_{K}$ we have

$$
\operatorname{Im}\left(\tilde{f}_{j}(x, y)\right) \leq \operatorname{Im}\left(\tilde{f}_{j}(0, y)\right)+2 K a_{j+1}=\tilde{g}_{j}(y)+2 K a_{j+1} \leq G_{K}(y)+2 K a_{j+1} .
$$

Now, (beside the already fixed $q$ ) we fix a point $p \in U_{0}$ and its lift $\tilde{p} \in \mathbb{C}$. For $j \geq 0$, we set

$$
\tilde{p}_{j}:=\tilde{f}^{j}(\tilde{p}), \quad z_{j}:=\psi_{j}^{-1}\left(\tilde{p}_{j}\right), \quad y_{j}:=\operatorname{Im}\left(z_{j}\right) .
$$

We shall use that $\rho\left(G_{K}\right)=0$ and $a_{j}$ 's are small to secure the following key claim.

Claim 6.1. There is $C=C\left(K, \mathrm{q}_{*}\right)>0$ with $\lim _{\mathbf{q}_{*} \rightarrow \infty} C\left(K, \mathrm{q}_{*}\right)=0$ such that

$$
\limsup _{j \rightarrow \infty}\left|y_{j}\right| / j \leq C\left(K, \mathbf{q}_{*}\right) \text {. }
$$

Before proving the claim, let us see that Theorem 6.8 already follows via the following general lemma (again relegated to Section 7 ).

Lemma 6.4 (Isthmus Estimate). If $\psi: B_{a} \rightarrow \mathbb{C}$ is a conformal univalent map such that $\psi(z+i)=\psi(z)+i$, then, for any $z, w \in B_{a}$, we have

$$
|\operatorname{Im}(\psi(z))-\operatorname{Im}(\psi(w))| \leq|\operatorname{Im}(z)-\operatorname{Im}(w)|+2 \sqrt{a \cdot \text { Area }}+4
$$

where Area is the Euclidean area of the annulus $\psi\left(B_{a}\right) / \mathbb{Z}$ (i.e. of $\psi([0, a] \times[0,1])$ ).

Indeed, because the conformal mapping $\tilde{\psi}_{n}: \tilde{B}_{a_{n}} \rightarrow \tilde{U}_{n}$ sends 0 and $z_{n}$ to $\tilde{q}_{n}$ and $\tilde{p}_{n}$, correspondingly (and the area is bounded by that of $\mathbb{T}^{2}$ ), the lemma combines with Claim 6.1 to secure

$$
\limsup _{n \rightarrow \infty} \frac{\left|\operatorname{Im}\left(\tilde{p}_{n}\right)-\operatorname{Im}\left(\tilde{q}_{n}\right)\right|}{n} \leq \limsup _{n \rightarrow \infty} \frac{\left|\operatorname{Im}\left(z_{n}\right)\right|}{n} \leq C\left(K, \mathbf{q}_{*}\right) .
$$

Theorem 6.8 follows immediately by arbitrariness of $p \in U_{0}$ and Fact 6.2 .

\footnotetext{
${ }^{5}$ actually, uniformly Hölder
} 
Proof of Claim [6.1. We show only that $\lim \sup _{j \rightarrow \infty} y_{j} / j \leq C\left(K, \mathbf{q}_{*}\right)$ because the other inequality, $\lim \sup _{j \rightarrow \infty}-y_{j} / j \leq C\left(K, \mathbf{q}_{*}\right)$, follows by reversing the $y$-axis.

Clearly $y_{j+1}=\operatorname{Im}\left(\tilde{f}_{j}\left(x_{j}+i y_{j}\right)\right)$, so Fact 6.5 yields

$$
y_{j+1} \leq G_{K}\left(y_{j}\right)+2 K a_{j+1}, \quad j \geq 0 .
$$

Thus, if we define $\left(t_{j}\right)_{j=0}^{\infty}$ recursively by

$$
t_{j+1}:=G_{K}\left(t_{j}\right)+2 K a_{j+1}, \quad t_{0}=y_{0},
$$

then the monotonicity of $G_{K}$ implies that

$$
y_{j} \leq t_{j}, \quad j \geq 0
$$

It suffices then to estimate the $t_{j}$ 's.

Since $a_{j} \leq 1$ and the Poincaré rotation number is a monotonic function of the circle homeomorphism (see e.g. [2]), we have a trivial bound $\lim \sup _{j \rightarrow \infty} t_{j} / j \leq$ $\rho\left(G_{K}\right)+2 K$. To improve the estimate, we shall use Fact 6.3 Fix an arbitrary $\nu>0$. From Fact 6.3 for any $k \in \mathbb{N}$, we estimate à la Chebyschev's inequality:

$$
\begin{gathered}
\#\left\{j: a_{k+j} \geq 8 / \nu \mathbf{q}_{*}, 0 \leq j<\mathbf{q}_{*}\right\} \cdot 8 / \nu \mathbf{q}_{*} \leq \sum_{j=0,} \sum_{a_{k+j} \geq 8 / \nu \mathbf{q}_{*}}^{\mathbf{q}_{*}-1} a_{k+j} \leq 8, \\
\#\left\{j: a_{k+j}<8 / \nu \mathbf{q}_{*}, 0 \leq j<\mathbf{q}_{*}\right\} \geq(1-\nu) \mathbf{q}_{*} .
\end{gathered}
$$

For any $n \in \mathbb{N}$, by dividing $\{1, \ldots, n\}$ into blocks of length $\mathbf{q}_{*}$, we conclude

$$
\#\left\{j: a_{j} \geq 8 / \nu \mathbf{q}_{*}, 0 \leq j<n\right\} \leq \nu\left(n / \mathbf{q}_{*}+1\right) \mathbf{q}_{*} .
$$

Let $M \subset \mathbb{N}$ be the set of indices for which $a_{k}$ is small,

$$
M:=\left\{k: a_{k} \leq 8 / \nu \mathbf{q}_{*}\right\} .
$$

For $k \in M$, set

$$
I_{k}:=\left\{k, k+1, \ldots, k+\left\lfloor\frac{\ln \mathrm{q}_{*}}{2 \ln K}\right\rfloor\right\},
$$

where $\lfloor\cdot\rfloor$ stands for the integer part. Because the modulus is a quasi-invariant and $f_{j}$ is $K$-quasiconformal, we have

$$
a_{j+1} \leq K a_{j} .
$$

By applying this $\left\lfloor\frac{\ln \mathrm{q}_{*}}{2 \ln K}\right\rfloor$ times, we get for $k \in M$ that

$$
a_{j} \leq \frac{8}{\nu \sqrt{\mathbf{q}_{*}}} \quad \text { for all } j \in I_{k} .
$$

The definition (6.3) of $t_{j}$ 's implies then that

$$
t_{j+1} \leq G_{K}\left(t_{j}\right)+\delta, \quad \delta:=2 K \cdot K \cdot \frac{8}{\nu \sqrt{\mathrm{q}_{*}}}=\frac{16 K^{2}}{\nu \sqrt{\mathrm{q}_{*}}}, \quad j \in I_{k} .
$$

Now, write

$$
\bigcup_{k \in M} I_{k}=S_{0} \cup S_{1} \cup S_{2} \cup \ldots
$$


where $S_{s}, s \geq 0$, are contiguous segments (i.e. $S_{s}=\left\{A_{s}, A_{s}+1, \ldots, B_{s}-1, B_{s}\right\}$ for some $A_{s}, B_{s} \in \mathbb{N}$ ). For $j \in S_{s}$, we have (6.7), so the standard estimate of displacement via the rotation number $\rho\left(G_{K}+\delta\right)$ yields

$$
t_{\max S_{s}}-t_{\min S_{s}} \leq \rho\left(G_{K}+\delta\right) \cdot\left(\max S_{s}-\min S_{s}\right)+1 .
$$

On the other hand, for $j \notin S_{s}$, taking into account that $a_{j} \leq 1$, we have a trivial bound

$$
t_{j+1}-t_{j} \leq \max _{y \in \mathbb{R}}\left(G_{K}(y)-y\right)+2 K .
$$

To combine the two estimates, for an arbitrary $n \in \mathbb{N}$, we note that the cardinality of the $S_{s}$ 's is at least $\left\lfloor\ln \mathrm{q}_{*} /(2 \ln K)\right\rfloor+1$, which guarantees that there is $l \leq n /\left(\left\lfloor\ln \mathrm{q}_{*} /(2 \ln K)\right\rfloor+1\right)$ such that $\max S_{l} \geq n$. Assuming for specificity that $n \in M$ (so that $n \in S_{l}$ ), we estimate

$$
\begin{gathered}
t_{n+1}-t_{0}=\sum_{j=0}^{\min S_{0}-1} t_{j+1}-t_{j}+\sum_{j=\min S_{0}}^{\max S_{0}} t_{j+1}-t_{j}+\sum_{j=\max S_{0}+1}^{\min S_{1}-1} t_{j+1}-t_{j} \\
\quad+\sum_{j=\min S_{1}}^{\max S_{1}} t_{j+1}-t_{j}+\ldots+\sum_{j=\min S_{l}}^{n} t_{j+1}-t_{j} \\
\leq \#\left\{j: 0 \leq j \leq n, j \notin S_{0} \cup S_{1} \cup S_{2} \cup \ldots\right\} \cdot \max _{y \in \mathbb{R}}\left(G_{K}(y)-y+2 K\right) \\
+\left(\# S_{0}+\# S_{1}+\ldots+\# S_{l-1}+n-\min S_{l}\right) \cdot \rho\left(G_{K}+\delta\right)+l \\
\leq \nu \cdot n\left(n / \mathbf{q}_{*}+1\right) \mathbf{q}_{*} \max _{y}\left(G_{K}(y)-y+2 K\right)+n \cdot \rho\left(G_{K}+\delta\right)+n \cdot\left(\left\lfloor\frac{\ln \mathbf{q}_{*}}{2 \ln K}\right\rfloor+1\right)^{-1}
\end{gathered}
$$

where we used (6.6) to estimate the cardinality in the first term. A very similar estimate holds when $n \notin M$, and one concludes that

$$
\begin{aligned}
\limsup _{n \rightarrow \infty} \frac{y_{n}}{n} & \leq \limsup _{n \rightarrow \infty} \frac{t_{n}}{n} \\
& \leq \nu\left(\max _{y}\left(G_{K}(y)-y\right)+2 K\right)+\rho\left(G_{K}+\delta\right)+\left(\left\lfloor\frac{\ln \mathbf{q}_{*}}{2 \ln K}\right\rfloor+1\right)^{-1} .
\end{aligned}
$$

Taking $\nu:=1 / \ln \mathrm{q}_{*}$, we claim that the right hand side of the last inequality constitutes the sought after bound $C\left(K, \mathbf{q}_{*}\right)$. This follows readily because $\rho\left(G_{K}\right)=0$ and $\delta \mapsto \rho\left(G_{K}+\delta\right)$ is continuous (since the rotation number is a continuous function of a circle map).

\section{Extremal Length Estimates}

We supply now the proofs of the general lemmas invoked in the previous section for the proof of Theorem 6.8 The common feature of all the arguments is their dependence on the extremal length 11. Recall that if $\Gamma$ is a family of rectifiable curves in $\mathbb{C}$ then the extremal length of $\Gamma$ is

$$
\lambda(\Gamma):=\sup \left\{L_{\Gamma}(\rho)^{2} / A(\rho): \rho: M \rightarrow[0, \infty) \text { measurable with } A(\rho) \neq 0, \infty\right\}
$$

where

$$
L_{\Gamma}(\rho):=\inf \left\{L_{\gamma}(\rho)=\int_{\gamma} \rho|d z|: \gamma \in \Gamma\right\} \quad \text { and } \quad A(\rho):=\iint \rho^{2} d x d y
$$


(Here, $|d z|=\sqrt{d x^{2}+d y^{2}}$.) In the more general case when $\Gamma$ is contained in a Riemannian surface, one has to replace $|d z|$ and $d x d y$ by the length and area elements of the Riemannian metric. The extremal length is a conformal invariant and often can be readily computed. For instance, if $\Gamma$ is a family of all rectifiable loops of homotopy type $(1,0)$ in the flat torus $\mathbb{T}^{2}=\mathbb{R}^{2} / \mathbb{Z}^{2} \equiv \mathbb{C} /(\mathbb{Z}+i \mathbb{Z})$, then $\lambda(\Gamma)=1$ from the Grötzsch inequality (see [1]).

Proof of Lemma 6.1. Our proof is an extension of the standard argument for superadditivity of extremal length as found in 1. (Application of Jensen's inequality and a bit different choice of $\rho$ below are the only novel elements.)

Let $\left\{V_{j}\right\}_{j=1}^{N}$ be annuli of homology type $(0,1)$ in $\mathbb{T}^{2}$ with the $m$-overlap property, meaning that there are $m$ essentially disjoint families of $(0,1)$-annuli, $\left\{V_{j}^{l}\right\}_{j=1}^{N}$, $l=1, \ldots, m$, such that $V_{j}=\bigcup_{l=1}^{m} V_{j}^{l}$ is an essentially disjoint sum for each $j=$ $1, \ldots, N$. The goal is to see that $\sum_{j=1}^{N} \bmod \left(V_{j}\right) \leq m^{3}$. If $\Gamma_{j}$ is to denote the family of all rectifiable arcs in $V_{j}$ connecting the two boundary components of $V_{j}$, then the modulus $a_{j}$ of $V_{j}$ is given by

$$
a_{j}=\lambda\left(\Gamma_{j}\right)
$$

Consider arbitrary measurable $\rho_{j}: V_{j} \rightarrow[0, \infty)$ with $A\left(\rho_{j}\right) \neq 0, \infty$ and $L_{\Gamma_{j}}\left(\rho_{j}\right)>$ 0 for $j=1, \ldots, N$. We shall think of $\rho_{j}$ as extended to all of $\mathbb{T}^{2}$ by zero. Since multiplication of $\rho_{j}$ by a constant does not affect $L_{\Gamma_{j}}\left(\rho_{j}\right)^{2} / A\left(\rho_{j}\right)$, we may assume that $A\left(\rho_{j}\right)=L_{\Gamma_{j}}\left(\rho_{j}\right)$. Let $\Gamma$ be a family of all rectifiable loops of homotopy type $(1,0)$ on $\mathbb{T}^{2}$. Note that, given $\gamma \in \Gamma$ and $1 \leq j \leq N$, we have $\gamma_{j} \subset \gamma \cap V_{j}$ that belongs to $\Gamma_{j}$ because $\gamma$ has to cut across $V_{j}$ for topological reasons. Set

$$
\rho:=\sum_{j=1}^{N} \rho_{j}, \quad \rho^{(l)}:=\sum_{j=1}^{N} \rho_{j} \chi_{V_{j}^{l}}, \quad \text { for } 1 \leq l \leq m,
$$

where $\chi_{A}$ stands for the characteristic function of the set $A$. Clearly,

$$
\rho=\sum_{l=1}^{m} \rho^{(l)} \text { a.e. } \quad \text { and } \quad \rho \geq \max _{1 \leq j \leq N} \rho_{j} .
$$

For any $\gamma \in \Gamma$, the essential disjointness hypotheses imply

$$
\begin{aligned}
m \cdot L_{\gamma}(\rho) \geq \sum_{l=1}^{m} \sum_{j=1}^{N} L_{\gamma \cap V_{j}^{l}}(\rho) & =\sum_{j=1}^{N} L_{\gamma \cap V_{j}}(\rho) \geq \sum_{j=1}^{N} L_{\gamma_{j}}(\rho) \\
& \geq \sum_{j=1}^{N} L_{\gamma_{j}}\left(\rho_{j}\right) \geq \sum_{j=1}^{N} L_{\Gamma_{j}}\left(\rho_{j}\right) .
\end{aligned}
$$

Therefore, the arbitrariness of $\gamma \in \Gamma$ yields

$$
m \cdot L_{\Gamma}(\rho) \geq \sum_{j=1}^{N} L_{\Gamma_{j}}\left(\rho_{j}\right)
$$


Also, from the essential disjointness of $\left\{V_{j}^{l}\right\}_{j=1}^{N}$, for each $l=1, \ldots, m,\left(\rho^{(l)}\right)^{2}=$ $\sum_{j=1}^{N} \rho_{j}^{2} \chi_{V_{j}^{l}}^{2}$ a.e., and we can use the convexity of $x \mapsto x^{2}$ to estimate as follows

$$
\begin{aligned}
\sum_{j=1}^{N} A\left(\rho_{j}\right)=\sum_{j=1}^{N} & \iint \rho_{j}^{2} \chi_{V_{j}}=\sum_{j=1}^{N} \sum_{l=1}^{m} \iint \rho_{j}^{2} \chi_{V_{j}^{l}} \\
& =\iint \sum_{l=1}^{m}\left(\rho^{(l)}\right)^{2} \geq 1 / m \cdot \iint\left(\sum_{l=1}^{m} \rho^{(l)}\right)^{2}=A(\rho) / m .
\end{aligned}
$$

The combined inequalities (7.1) and (7.2) yield

$$
\lambda(\Gamma) \geq L_{\Gamma}(\rho)^{2} / A(\rho) \geq \frac{\left(\sum_{j=1}^{N} L_{\Gamma_{j}}\left(\rho_{j}\right) / m\right)^{2}}{m \cdot \sum_{j=1}^{N} A\left(\rho_{j}\right)}=1 / m^{3} \cdot\left(\sum_{j=1}^{N} L_{\Gamma_{j}}\left(\rho_{j}\right)\right)
$$

where the last equality uses the normalization $L_{\Gamma_{j}}\left(\rho_{j}\right)=A\left(\rho_{j}\right)$. The arbitrariness of $\rho_{j}$ 's yields

$$
\lambda(\Gamma) \geq 1 / m^{3} \cdot\left(\sum_{j=1}^{N} \lambda\left(\Gamma_{j}\right)\right) .
$$

Because $\lambda(\Gamma)=1$, we are done.

Proof of Lemma 6.3. Let $0<a, b<1$ and $\phi: B_{a} \rightarrow B_{b}$ be a $K$-quasiconformal homeomorphism. Fix $z=x+i y \in B_{a}$ and set $y_{0}:=\operatorname{Im}(\phi(0+i y))$ and $y_{1}:=$ $\operatorname{Im}(\phi(x+i y))$. To show the lemma we have to argue that $\left|y_{1}-y_{0}\right| \leq 2 K b$.
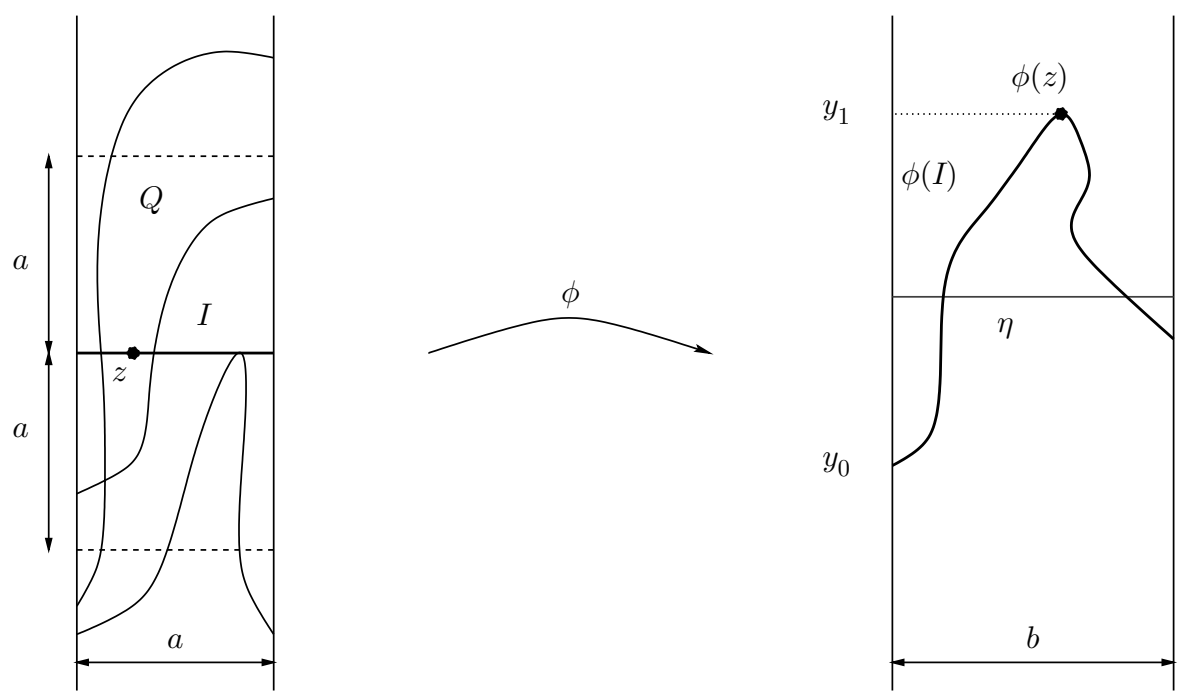

Figure 7.1. Shear Estimate. (Some example curves from $\Gamma$ are pictured on the left.) 
Consider the family $\Gamma$ of all rectifiable curves traversing $B_{a}$ and hitting the horizontal segment $I:=\left\{z \in B_{a}: \operatorname{Im}(z)=y\right\}$; namely,

$$
\begin{array}{r}
\Gamma:=\left\{\gamma:[0,1] \rightarrow B_{a}: \operatorname{Re}(\gamma(0))=0, \operatorname{Re}(\gamma(1))=a,\right. \\
\operatorname{Im}(\gamma(t))=y \text { for some } t \in[0,1]\} .
\end{array}
$$

Every horizontal segment in the family

$$
\Gamma_{1}=\left\{\eta:[0,1] \rightarrow B_{b}: \eta(t)=t b+i c, c \in\left[y_{0}, y_{1}\right]\right\}
$$

clearly hits $\phi(I)$, which means that $\Gamma_{1} \subset \phi(\Gamma)$. By the quasi-invariance of extremal length, monotonicity of extremal length, and the Grötzsch formula for the rectangle's modulus (in that order), we have

$$
\lambda(\Gamma) / K \leq \lambda(\phi(\Gamma)) \leq \lambda\left(\Gamma_{1}\right)=b /\left|y_{1}-y_{0}\right| .
$$

To estimate $\lambda(\Gamma)$, we take $\rho: B_{a} \rightarrow[0, \infty)$ to be the characteristic function of the rectangle $Q:=\left\{z \in B_{a}: \operatorname{Im}(z) \in[y-a, y+a]\right\}$. Clearly, $A(\rho)=2 a^{2}$. Moreover, for any $\gamma \in \Gamma$, the geometry (see Figure 7.1) forces the length of $\gamma \cap Q$ to be at least $a$, i.e. $L_{\gamma}(\rho) \geq L_{\gamma \cap Q}(1) \geq a$. Hence,

$$
\lambda(\Gamma) \geq a^{2} /\left(2 a^{2}\right)=1 / 2 .
$$

Putting together the two displayed inequalities yields the desired bound:

$$
1 / 2 \leq \lambda(\Gamma) \leq K \cdot \lambda(\phi(\Gamma)) \leq K \cdot \lambda\left(\Gamma_{1}\right)=K b /\left|y_{1}-y_{0}\right| \cdot
$$

Proof of Lemma 6.2 This is a variant of Mori's theorem (see Theorem 4.1 in Chapter II of [24). Denote $Q_{a}:=\left\{z \in B_{a}: 0 \leq \operatorname{Im}(z) \leq 1\right\}$. The restrictions of maps in $\mathcal{F}_{K}$ to the fundamental domain $Q_{a}$ belong to the family $\left\{\phi: Q_{a} \rightarrow\right.$ $B_{b} K$-q.c. homeo. onto image, $\left.a, b<1\right\}$, which is equicontinuous with respect to the spherical metric on the force of Mori's theorem because the range of each such $\phi$ clearly omits two points of definite spherical distance (take e.g. 3 and $3+i$ ). The equicontinuity of $\mathcal{F}^{\mathbb{C}}{ }_{K}$ with respect to the Euclidean metric on $\mathbb{C}$ will follow if we find $R>0$ such that $\phi\left(Q_{a}\right) \subset\{|z| \leq R\}$ for all $\phi \in \mathcal{F}^{\mathbb{C}}{ }_{K}$.

For $\phi \in \mathcal{F}^{\mathbb{C}}{ }_{K}, \phi(0)=0$ and $\phi(i)=i$ so that $0 \leq \operatorname{Im}(\phi(i y)) \leq 1$ for all $0 \leq y \leq 1$. Lemma 6.3 implies then that $-2 K b \leq \operatorname{Im}(\phi(x+i y)) \leq 1+2 K b$ for any $x+i y \in Q_{a}$. Therefore, $R=\sqrt{(1+2 K)^{2}+1^{2}}$ is as required.

Proof of Lemma 6.4 Recall that $\psi: B_{a} \rightarrow \mathbb{C}$ is conformal, univalent, and equivariant: $\psi(z+i)=\psi(z)+i$ for $z \in B_{a}$. Let $\Gamma_{0}$ be the family of horizontal segments cutting across the fundamental domain $Q=\left\{z \in B_{a}: 0 \leq \operatorname{Im}(z) \leq 1\right\}$,

$$
\Gamma_{0}=\left\{\eta:[0,1] \rightarrow B_{a}: \eta(t)=t a+i c, c \in[0,1]\right\}, \quad \lambda\left(\Gamma_{0}\right)=a .
$$

For $\Gamma:=\psi\left(\Gamma_{0}\right)$, we have $\lambda(\Gamma)=\lambda\left(\Gamma_{0}\right)=a$ because $\psi$ is conformal. By taking the metric on the annulus $\psi\left(B_{a}\right) / \mathbb{Z}$ induced by the standard metric on $\mathbb{C}$, the definition of $\lambda(\Gamma)$ yields

$$
\inf _{\gamma \in \Gamma} L(\gamma)^{2} / A(\psi(Q)) \leq \lambda(\Gamma)=a .
$$

We can then find $\gamma \in \Gamma$ of length $L(\gamma) \leq \sqrt{a \cdot A(\psi(Q))}$.

Fix now $w, z \in B_{a}$. For any $k \in \mathbb{Z}$, the quadrilateral bounded by $\gamma+k i$ and $\gamma+(k+1) i$ in $\psi\left(B_{a}\right)$ is a fundamental domain; therefore, it contains $\phi(z)$ for some $k$ (which we fix). The vertical line through $\psi(z)$ hits $\gamma+k i$ at some point 
$p \in \gamma+k i$ with $|p-\psi(z)| \leq 1$. Analogously, $l \in \mathbb{Z}$ and $q \in \gamma+l$ can be found with $|q-\psi(w)| \leq 1$. By the triangle inequality,

$$
|\operatorname{Im}(\psi(z))-\operatorname{Im}(\psi(w))| \leq|\psi(z)-\psi(w)| \leq|p-q|+2 .
$$

Furthermore, the length estimate on $\gamma$ and the triangle inequality again give

$$
|p-q| \leq|k-l| \cdot 1+2 \cdot \operatorname{diam}(\gamma) \leq|k-l|+2 \sqrt{a \cdot A(\psi(Q))} .
$$

Similarly, since $\psi^{-1}(\gamma)$ is a horizontal segment, we have

$$
|\operatorname{Im}(z)-\operatorname{Im}(w)| \geq\left|\operatorname{Im}\left(\psi^{-1}(p)\right)-\operatorname{Im}\left(\psi^{-1}(q)\right)\right|-2=|k-l|-2 .
$$

By putting the three inequalities together, we get the assertion of the lemma:

$$
|\operatorname{Im}(\psi(z))-\operatorname{Im}(\psi(w))| \leq|\operatorname{Im}(z)-\operatorname{Im}(w)|+2 \sqrt{a \cdot A(\psi(Q))}+4 .
$$

\section{Appendix: EmbedDings into $\mathbb{T}^{3}$}

The existence of a clean pair of cross-sections in Section 3 depended on two intuitively obvious facts: a sphere embedded in $\mathbb{T}^{3}$ bounds a topological 3-disk; a two-torus $\mathbb{T}^{2}$ embedded in $\mathbb{T}^{3}$ bounds a topological solid torus. These, however, are not easy to prove and require (cf. Alexander's horned sphere) hypothesis on regularity of the embeddings. For instance, it suffices to require that the embeddings are polyhedral or extend to bi-collar neighborhoods, which is the case for the piecewise smooth embeddings we encountered. Below we indicate how to deduce the results we used in Section 3 from readily available classical theorems.

Theorem 8.9 (Schönflies Theorem in $\mathbb{T}^{3}$ ). If $g: \mathbb{S}^{2} \rightarrow \mathbb{T}^{3}$ is a piecewise smooth embedding, then $M:=g\left(\mathbb{S}^{2}\right)$ bounds a topological 3-disk in $\mathbb{T}^{3}$ and $g$ extends to an embedding $G: \mathbb{B}^{3} \rightarrow \mathbb{T}^{3}$.

The reduction to the classical Schönflies Theorem 30 about embeddings into $\mathbb{R}^{3}$ will be afforded by the following simple lemma from general topology, the proof of which we leave as an exercise.

Lemma 8.5. Suppose $\pi$ is an open and locally injective mapping (between metric spaces). If $A$ is compact with $\partial A$ connected, $\partial \pi(A) \neq \emptyset$, and $\left.\pi\right|_{\partial A}$ is injective, then $\left.\pi\right|_{A}$ is injective.

Sketch of Proof of Theorem [8.9, Lift $g$ to $\tilde{g}: \mathbb{S}^{2} \rightarrow \mathbb{R}^{3}$. Smoothness assures that $\tilde{g}\left(\mathbb{S}^{2}\right)$ has a tubular neighborhood in $\mathbb{R}^{3}$, so that $\tilde{g}$ can be extended to an embedding $\mathbb{S}^{2} \times[-1,1] \rightarrow \mathbb{T}^{3}$ - we skip the details of this routine construction. By the classical Schönflies Theorem (as found in [4]), $\tilde{g}\left(\mathbb{S}^{2}\right)$ bounds a 3 -disk in $\mathbb{R}^{3}$ and $\tilde{g}$ extends to an embedding $\tilde{G}: \mathbb{B}^{3} \rightarrow \mathbb{R}^{3}$. The map $G:=\pi \circ \tilde{G}$ (where $\pi$ is the universal covering) is clearly an extension of $g$. That $G$ is an embedding follows from the lemme applied to $\left.\pi\right|_{\tilde{G}\left(\mathbb{B}^{3}\right)}$.

The following result is reminiscent of Alexander's theorem on the embeddings of $\mathbb{T}^{2}$ into $\mathbb{S}^{3}$.

Theorem 8.10 (Alexander's Theorem in $\mathbb{T}^{3}$ ). If $g: \mathbb{T}^{2} \rightarrow \mathbb{T}^{3}$ is a piecewise smooth embedding and the induced map on the first homology is of rank one, then $M:=$ $g\left(\mathbb{S}^{2}\right)$ bounds a solid 3-torus $B$ in $\mathbb{T}^{3}$ and $g$ extends to an embedding $G: \mathbb{B}^{2} \times \mathbb{T} \rightarrow$ $\mathbb{T}^{3}$. 


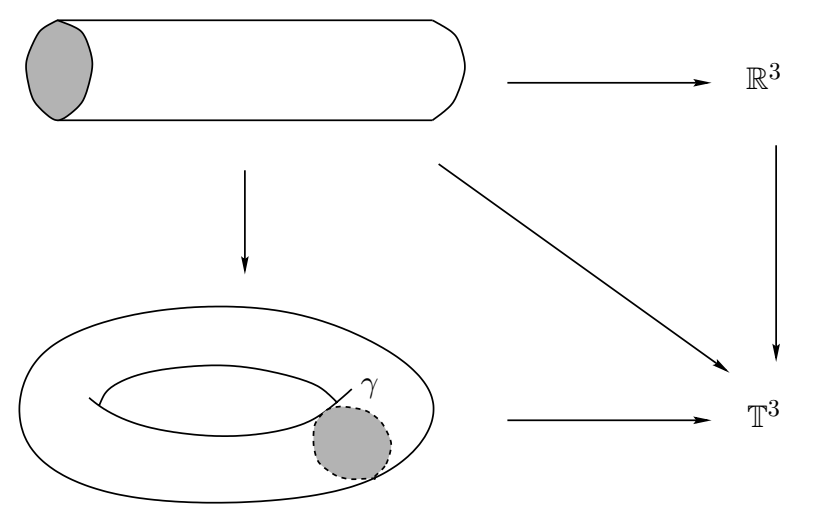

Figure 8.1.

Sketch of Proof of Theorem 8.10, No generality is lost in assuming that $H_{1}\left(\mathbb{T}^{2}\right)$ is mapped to $\operatorname{lin}\{(1,0,0)\}$ in $H_{1}\left(\mathbb{T}^{3}\right)$. (Recall, we use the standard identification of $H_{1}\left(\mathbb{T}^{3}\right)$ with $\mathbb{R}^{3}$.) As before, smoothness yields a nice tubular neighborhood of $g\left(\mathbb{T}^{2}\right)$ in $\mathbb{T}^{3}$; which (by PL approximation) assures that $g\left(\mathbb{T}^{2}\right)$ is polyhedral and two sided in $\mathbb{T}^{3}$ (see [30] for definitions). Since the kernel of the action induced by $g$ on the fundamental group is non-trivial, the Loop Theorem (see page 193 in [30]) supplies a (polyhedral) two-disk $\Delta$ in $\mathbb{T}^{3}$ with $\partial \Delta$ not contractible in $g\left(\mathbb{T}^{2}\right)$ and such that $\Delta \cap g\left(\mathbb{T}^{2}\right)=\partial \Delta$. Let $\delta: \mathbb{B}^{2} \rightarrow \mathbb{T}^{3}$ be the embedding onto $\delta\left(\mathbb{B}^{2}\right)=\Delta$. Now, cut $\mathbb{T}^{2}$ along the loop $\gamma:=g^{-1}(\partial \Delta)$ and cap each end of the resulting cylinder with a 2-disk as depicted below. Having identified the resulting two-sphere with $\mathbb{S}^{2}, g$ and two disjoint copies of $\delta$ combine to form an immersion $g_{1}: \mathbb{S}^{2} \rightarrow \mathbb{T}^{3}$. The lift $\tilde{g}_{1}: \mathbb{S}^{2} \rightarrow \mathbb{R}^{3}$ of $g_{1}$ is an embedding and extends to an embedding $\tilde{G}_{1}: \mathbb{B}^{3} \rightarrow \mathbb{R}^{3}$ by Schönflies Theorem (the polyhedral version found in [30]). Now, the natural identification of the two-disks on $\mathbb{S}^{2}$ yields a solid torus $B$; and the map $\tilde{G}_{1}$ descends to $G: B \rightarrow \mathbb{T}^{3}$ that extends $g$. It is not difficult to see that $G$ is an embedding.

Finally, let us show the following lemma employed in Section 4 to straighten the cross-sections.

Lemma 8.6 (Straightening). Given two smoothly embedded two-tori in $\mathbb{T}^{3}$ that intersect transversally along a simple loop, there is a homotopic to the identity diffeomorphism of $\mathbb{T}^{3}$ that maps each of the two-tori to a flat torus (i.e. a torus obtained as a quotient of a sub-plane in $\mathbb{R}^{3}$ ).

Sketch of Proof of Lemma 8.6. We fix two copies of a 2-torus, $\mathbb{T}^{2}$ and $\mathbb{T}_{*}^{2}$, and think of them as the flat sub-tori in $\mathbb{T}^{3}$ of cohomology type $(0,0,1)$ and $(1,0,0)$, respectively.

Let $\sigma: \mathbb{T}^{2} \rightarrow \mathbb{T}^{3}$ and $\sigma_{*}: \mathbb{T}_{*}^{2} \rightarrow \mathbb{T}^{3}$ be the two smooth embeddings in the lemma, and set $\Sigma=\sigma\left(\mathbb{T}^{2}\right)$ and $\Sigma_{*}^{*}=\sigma\left(\mathbb{T}_{*}^{2}\right)$. Since the intersection product of $\Sigma$ and $\Sigma_{*}$ is a class of a simple loop (cf. Section 3), $\Sigma$ and $\Sigma_{*}$ must be in different cohomology classes. For simplicity, let us assume that $\Sigma$ is cohomologous to $\mathbb{T}^{2}$ and $\Sigma_{*}$ is cohomologous to $\mathbb{T}_{*}^{2}$, as this can always be achieved by post-composing the embeddings with an appropriate automorphism of $\mathbb{T}^{3}$. Therefore, $\Gamma:=\Sigma \cap \Sigma_{*}$ is a 


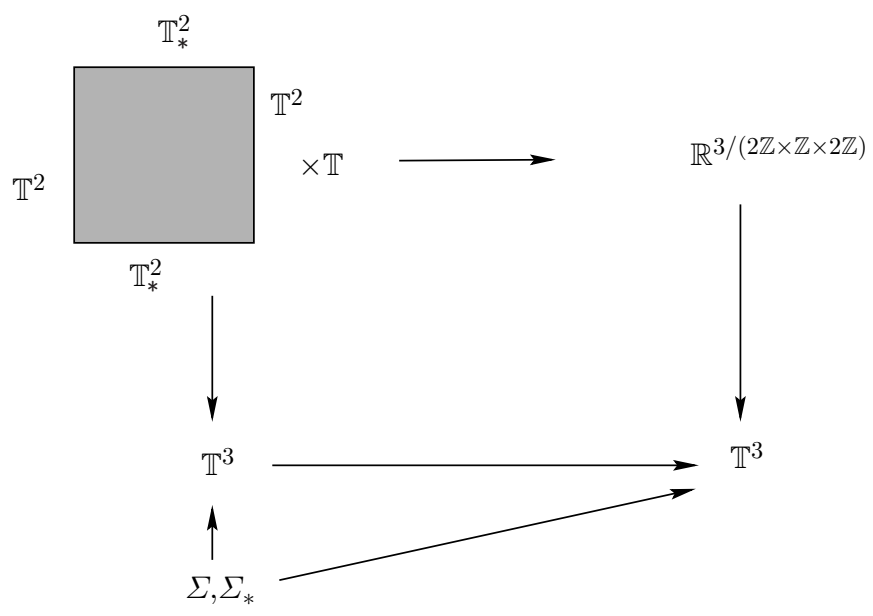

Figure 8.2.

$(0,1,0)$ loop in $\mathbb{T}^{3}$. It can be arranged 6 that $\alpha:=\sigma^{-1}(\Gamma)$ and $\alpha_{*}:=\sigma_{*}{ }^{-1}(\Gamma)$ are $(0,1)$ loops. Now, take two copies of $\mathbb{T}^{2}$ each sliced along $\alpha$, and two copies of $\mathbb{T}_{*}^{2}$ each sliced along $\alpha_{*}$; and put together the four resulting annuli to form a torus $M$ as hinted by Figure 8.2

The embeddings $\sigma$ and $\sigma_{*}$ naturally induce $f: M \rightarrow \mathbb{T}^{3}$ and its lift $\tilde{f}: M \rightarrow$ $\mathbb{R}^{3} /(2 \mathbb{Z} \times \mathbb{Z} \times 2 \mathbb{Z}$ ) to a bigger torus is an embedding (unlike $f$ ). By Theorem 8.10 , $\tilde{f}$ extends to the solid torus $B$ bounded by $M$. This extension factors through the natural identifications on $M=\partial B$ to yield some $F_{1}: B / \sim \cong \mathbb{T}^{3} \rightarrow \mathbb{R}^{3} /(2 \mathbb{Z} \times$ $\mathbb{Z} \times 2 \mathbb{Z})$. From the construction, $F_{1}$ is easily seen to descend to an embedding $F: \mathbb{T}^{3} \rightarrow \mathbb{T}^{3}$ that extends $\sigma$ and $\sigma_{*}$. Equally easy is to see that $F$ induces the identity on the first homology and therefore must be homotopic to the identity.

\section{REFERENCES}

1. L. Ahlfors, Lectures on Quasiconformal Mappings, Van Nostrand, New York, 1966. MR 34:336

2. L. Alseda, J. Llibre, and M. Misiurewicz, Combinatorial Dynamics and Entropy in Dimension One, Advanced Series in Nonlinear Dynamics, vol. 5, World Scientific, Singapore, 1993. MR 95j:58042

3. C. Baesens, J. Guckenheimer, S. Kim, and R. MacKay, Three coupled oscillators: modelocking, global bifurcations and toroidal chaos, Physica D 49 (1991), 387-475. MR 93c:58155

4. G. E. Bredon, Topology and geometry, Springer, New York, 1993.

5. P. Le Calvez, Propriétés dynamiques des difféomorphismes de l'anneau et du tore, Astérisque, vol. 204, 1991. MR 94d:58092

6. J. Franks, Realizing rotation vectors for torus homeomorphisms, Trans. Amer. Math. Soc. 311 (1989), 107-115. MR 89k:58239

7. (1992), 217-226. MR 93m:58059

8. and chaos, Vol. 1, World Sci. Publishing, River Edge, NJ, 1995, p. 41. MR 98j:58092

9. - Rotation vectors and fixed points of area preserving surface diffeomorphisms, Trans. Amer. Math. Soc. 348 (1996), no. 7, 2637-2662. MR 96i:58143

10. J. Franks and M. Misiurewicz, Rotation sets of toral flows, Proc. Amer. Math. Soc. 109 (1990), 243-249. MR 90i:58091

\footnotetext{
${ }^{6}$ by precomposing the embeddings with an automorphism of $\mathbb{T}^{2}$
} 
11. D. Fried, The geometry of cross sections to flows, Topology 21 (1982), 353-371. MR 84d:58068

12. M. Handel, Periodic point free homeomorphisms of $\mathbf{T}^{2}$, Proc. Amer. Math. Soc. 107 (1989), 511-515. MR 90c:58168

13. M. R. Herman, Une méthode pour minorer les exposants de Lyapounov et quelques exemples montrant le caractère local d'un théorème d'Arnold et de Moser sur le tore de dimension 2, Comment. Math. Helv. 58 (1983), 453-502. MR 85g:58057

14. Some open problems in dynamical systems, 1998, Doc. Math. Extra Volume II, ICM Proc. Internat. Congr. Math., vol. II (Berlin, 1998), 797-808. MR 99i:58047

15. R. A. Johnson, Ergodic theory and linear differential equations, J. Differential Equations 28 no. 1 (1978), 23-34. MR 80c:34044

16. R. A. Johnson and J. Moser, The rotation number for almost periodic potentials, Comm. in Math. Phys. 84 (1982), 403-438. MR 83h:34018

17. L.B. Jonker and L. Zhang, Torus homeomorphisms whose rotation sets have empty interior, Erg. Th. \& Dyn. Sys. 18 (1998), 1173-1185. MR 99h:58147

18. J. Kwapisz, Every convex rational polygon is a rotation set, Erg. Th. \& Dyn. Sys. 12 (1992), 333-339. MR 93g:58082

19. _ Rotation sets and entropy, SUNY at Stony Brook PhD thesis, 1995.

20. - A toral diffeomorphism with a non-polygonal rotation set, Nonlinearity 8 (1995), 461-476. MR 96j:58099

21. Nonlinearity 13 (2000), 1841-1854. MR 2001h:37089

22. — Combinatorics of torus diffeomorphisms, to be published in Erg. Th. \& Dyn. Sys., 2001.

23. - Renormalizing torus and annulus maps, in progress, 2001.

24. O. Lehto and K.I. Virtanen, Quasiconformal Mappings in the Plane, Springer, New York, 1973. MR 49:9202

25. J. Llibre and R. MacKay, Rotation vectors and entropy for homeomorphisms of the torus isotopic to the identity, Erg. Th. \& Dyn. Sys. 11 (1991), 115-128. MR 92b:58184

26. R. Mañé, Ergodic theory and differentiable dynamics, Springer, Berlin, 1987. MR 88c:58040

27. D. McDuff and D. Salamon, Introduction to symplectic topology, Oxford Science Publications, New York, 1998. MR 2000g:53098

28. M. Misiurewicz and K. Ziemian, Rotation sets for maps of tori, J. London Math. Soc. 40 (1989), 490-506. MR 91f:58052

29. — Rotation sets and ergodic measures for torus homeomorphisms, Fund. Math. 137 (1991), 45-52. MR 92d:58106

30. E. E. Moise, Geometric topology in dimmensions 2 and 3, Springer, New York, 1977. MR 58:7631

31. E. Slaminka, A Brouwer translation theorem for free homeomorphisms, Trans. Amer. Math. Soc. 277 (1988), 277-291. MR 89c:54081

32. V. V. Veremenyuk, The existence of the rotation number for the equation $x^{\prime}=\lambda(t, x)$ with a right-hand side that is periodic in $x$ and almost periodic in $t$, Differentsialnye Uravneniya 27 No 6 (1991), 1073-1076, in Russian. MR 92h:34098

Department of Mathematical Sciences, Montana State University, Bozeman, MonTANA 59717-2400

E-mail address: jarek@math.montana.edu 